TITLE:

\title{
Generalization of the classical method for calculating dynamic hysteresis loops in grain-oriented electrical steels
}

\section{$\operatorname{AUTHOR}(\mathrm{S}):$}

Zirka, Sergey E.; Moroz, Yuri I.; Marketos, Philip; Moses, Anthony J.; Jiles, David C.; Matsuo, T.

\section{CITATION:}

Zirka, Sergey E. ...[et al]. Generalization of the classical method for calculating dynamic hysteresis loops in grain-oriented electrical steels. IEEE TRANSACTIONS ON MAGNETICS 2008, 44(9): 2113-2126

\section{ISSUE DATE:}

2008-09

URL:

http://hdl.handle.net/2433/84541

\section{RIGHT:}

(c) 2008 IEEE. Personal use of this material is permitted. However, permission to reprint/republish this material for advertising or promotional purposes or for creating new collective works for resale or redistribution to servers or lists, or to reuse any copyrighted component of this work in other works must be obtained from the IEEE. 


\title{
Generalization of the Classical Method for Calculating Dynamic Hysteresis Loops in Grain-Oriented Electrical Steels
}

\author{
Sergey E. Zirka ${ }^{1}$, Yuri I. Moroz ${ }^{1}$, Philip Marketos ${ }^{2}$, Anthony J. Moses², David C. Jiles ${ }^{2}$, and T. Matsuo ${ }^{3}$ \\ ${ }^{1}$ Department of Applied Physics and Technology, Dnepropetrovsk National University, Dnepropetrovsk 49050, Ukraine \\ ${ }^{2}$ Wolfson Centre for Magnetics, School of Engineering, Cardiff University, Cardiff CF24 3AA, U.K. \\ ${ }^{3}$ Department of Electrical Engineering, Kyoto University, Kyoto 615-8510, Japan
}

\begin{abstract}
We have studied the ability of two one-dimensional (1-D) time-stepping models, both based on the concept of magnetic viscosity, to reproduce dynamic loops and losses in grain-oriented (GO) electrical steels under arbitrary magnetization regimes. We found that GO steels $(0.3 \mathrm{~mm}$ thick) can be modeled quite accurately at magnetizing frequencies up to $200 \mathrm{~Hz}$ by a thin sheet representation, which is applied to a bulk material. At higher frequencies, acceptable results can be obtained through a finite-difference solver of a 1-D penetration equation whose applicability to GO steels can be explained in terms of domain wall bowing. Because of the inertial effect introduced by the magnetic viscosity, the average error in the loss prediction is reduced from $40 \%$ for the conventional classical method to $5 \%$ for the methods we studied. We demonstrated the accuracy of the models using two GO steels whose losses and $B-H$ characteristics were measured by computer-controlled Epstein and single-sheet testers.
\end{abstract}

Index Terms-Finite-difference time-domain analysis, grain-oriented steel, losses, magnetic hysteresis.

\section{INTRODUCTION}

G RAIN-ORIENTED (GO) electrical steel is almost universally used for power transformer and converter cores due to its superior magnetic properties when magnetized along the strip rolling direction. Although the need for an accurate description of magnetization processes in GO steel has grown with its development, it remains until now a largely unsolved physical and engineering problem. From an engineering viewpoint, an adequate solution of the problem would be a model, which is able to predict details of dynamic magnetization curves (or $B-H$ loops) under arbitrary magnetization conditions and which can be incorporated into a transient simulator (electric circuit) where these conditions are not known in advance. Among a wide range of technical problems which could be solved with such a model, iron loss evaluation is perhaps the most important, especially in view of the wide variety of magnetization conditions encountered in modern applications. Although the prediction of the specific energy loss (that is the area enclosed by the dynamic hysteresis loop) is not a sufficient criterion of the adequacy of the model, the loss aspect is always at the focus of the consideration of more general problem of the loop shape prediction.

Historically, the first (classical) magnetodynamic model [1] of a thin ferromagnetic sheet was based on the solution of the one-dimensional (1-D) Maxwell equation for plane electromagnetic waves penetrating a homogeneous (nondomain) material through the sheet surfaces. The plane nature of the waves is illustrated in Fig. 1(a), where dashed lines show the imaginary surfaces (parallel to the surface of the sheet) each point of which is characterized by equal values of the magnetic induction $B$ and magnetic field $H$ (both normal to the sheet cross section shown in Fig. 1).

It was revealed long ago that the total loss calculated with this model is invariably underestimated, even if an accurate static

Digital Object Identifier 10.1109/TMAG.2008.2000662

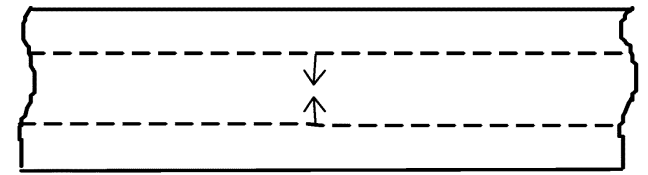

a)

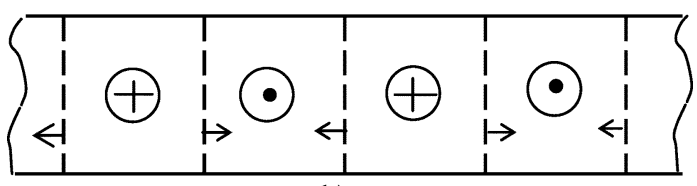

b)

Fig. 1. (a) Classical and (b) domain representations of the loss calculation problem in a thin sheet.

hysteresis model (SHM) is used to link the values of $B$ and $H$ in the 1-D Maxwell (penetration) equation. The difference between measured and calculated losses, the so-called anomalous or excess loss, becomes unacceptably large when the conventional classical approach is applied to GO steel, Permalloy, and many other commercial materials.

While the earlier explanations of the excess loss have been suggested in terms of a modification of the classical eddy-current theory (corresponding works are reviewed in [1]), this approach was later discarded in the hope of building a completely new magnetization model without using the excess loss concept. An alternative has been found in the framework of the domain approach according to which changes in magnetization are mainly confined within the thin domain walls (DWs), which separate moving bar domains alternately magnetized at positive and negative saturation. It is assumed that local electromotive forces (EMFs) induced by such a motion are the source of microscopic eddy currents and corresponding losses. The loss calculation in a regular domain structure of GO steel was initiated by the well-known model of Pry and Bean (PB) [2], where the motion of rigid DWs normal to the sheet surfaces, Fig. 1(b), was analyzed. Contrary to the 1-D classical model with its plane waves penetrating into the sheet depth, the 2-D PB model and its 
modifications are based on an array of magnetic domains whose rigid or bowing walls move parallel to the sheet surface.

Despite numerous attempts to improve the PB model (for a review, see [3]), its oversimplified nature was finally realized, and the loss calculation techniques based on the continuous motion of regular domain structures are accepted today as being of limited validity [4]. One reason for inaccuracy of the known DW models is that most of them disregard the rotation of the magnetization vector (domain rotation) which contributes considerably at high magnetic induction levels. They also ignore wall pinning on defects and different types of inhomogeneities distributed over the sheet cross section [5]. These pinning sites cause a discontinuous motion of domain walls or parts of walls up to the jumps characterized by the energy dissipated by relaxation damping [6], [7], [34], [35]. The energy loss can also be caused by the magnetostrictive deformation within DWs. According to [6], [34], and [35], this friction type mechanism is responsible for the excess loss and a substantial part of the hysteresis loss. The main part of hysteresis phenomena may be explained by DW pinning that gives rise to a friction force opposing the wall motion [8]. The situation is further complicated if we take into account the finite length of domains, their different orientations in different grains, the presence of flux closure domains [9], the unequal speed of different DWs, and domain nucleation and annihilation [10].

The energy loss, apart from that caused by eddy currents, is due to phonon dissipation as a result of domain processes. Since these factors can participate simultaneously in the magnetization process [11], it is practically impossible to combine them within a single deterministic model. For this reason, it was found expedient to deal with the loss problem in the framework of a statistical approach and the loss separation principle [12], in which the total loss, $W$, is decomposed into the components, known as hysteresis loss, $W_{\mathrm{h}}$, classical eddy-current loss, $W_{\text {clas }}$, and excess loss, $W_{\mathrm{exc}}$. Although the use of the classical component is by no means obvious when the domain structure of Fig. 1(b) is assumed, the loss separation is widely employed for GO steel.

Another tool for inclusion of $W_{\text {exc }}$ in the loop and loss calculation is the dynamic Preisach model (DPM) [13] of hysteresis which can be used instead of SHM in two ways. The first is the application of the DPM to a specimen as a whole, and the second is its incorporation into a 1-D Maxwell solver where it is applied independently to every finite element in the sheet.

An attempt to apply a generalized DPM to bulk GO steel has been reported in [14] where its suitability at low sinusoidal inductions was shown up to $100 \mathrm{~Hz}$. At levels higher than $1.3 \mathrm{~T}$ the model [14] overestimates the excess loss in a way revealed in [15].

The latter (magnetodynamic) utilization of the DPM is widely implemented in modeling nonoriented (NO) steels [16]. However, we are not aware of any reports on such application of the DPM to GO steels, although it is likely that attempts have been made in this direction. This points at substantial difference between these materials and serious difficulties arising in the modeling of GO steels.

In this paper, an attempt is made to answer the question whether it is possible to develop a phenomenological model,
TABLE I

MEASURED PARAMETERS OF THE STEELS EMPLOYED

\begin{tabular}{|c|c|c|c|c|c|}
\hline Steels & $\begin{array}{c}\rho, \\
\mu \Omega \cdot \mathrm{m}\end{array}$ & $\begin{array}{c}d, \\
\mathrm{~mm}\end{array}$ & $\begin{array}{c}H_{\mathrm{c}}, \\
\mathrm{A} / \mathrm{m}\end{array}$ & $\begin{array}{c}B_{\mathrm{r}}, \\
\mathrm{T}\end{array}$ & $\begin{array}{c}\text { Measurement } \\
\text { fixture }\end{array}$ \\
\hline Steel 1 & 0.480 & 0.255 & 7.9 & 1.58 & Epstein \\
\hline Steel 2 & 0.500 & 0.300 & 9.0 & 1.55 & SST \\
\hline
\end{tabular}

which would be capable of satisfactorily predicting the shape of the dynamic hysteresis loop of a sheet of typical GO steel when the frequency of a sinusoidal (or the fundamental frequency of nonsinusoidal) excitation voltage lies within a limited frequency range, say within 0-200 Hz. It is assumed that several dynamic loops used for the model fitting are measured at sinusoidal induction within this range. It is also assumed that the frequency content of the nonsinusoidal voltage can be substantially wider than that used for sinusoidal measurements, so one cannot use "black-box" methods, such as artificial neural networks, which are essentially interpolative and do not possess extrapolating ability [17].

The shortcomings of the DPM revealed in [15] have led us to the idea of using the viscous-type dynamic hysteresis model (DHM) [18], which maintains a fixed dependence of the excess loss over an unlimited frequency range and provides the possibility of changing the shape of the dynamic hysteresis loop. The DHM has been incorporated in 1-D magnetodynamic model [19] and also used for the "bulk calculation" [20]. Both these applications of the DHM concerned NO steel sheets whose properties can be experimentally studied quite accurately using the Epstein frame [21]. In the present case of GO steel, the dynamic loops calculated with single sheet models can be somewhat different from those measured with Epstein frame, which should be considered in this case as 3-D fixture characterized by the flux deviation from the rolling direction in the corners and therefore a variation of the magnetic path length with the induction level [22]. For this reason, we have also used the data [23] taken from a single sheet tester (SST) whose advantage is a constant magnetic path. Although the data obtained with the Epstein frame and SST have been taken from different sample geometries and at different laboratories, the electromagnetic parameters of the GO steels investigated with these standardized fixtures are close (see Table I), thus giving an indirect comparison of the setups employed.

\section{EXPERIMENTATION}

Two GO steels, which are referred to as Steels 1 and 2, have been employed in the study. Their resistivity $\rho$, lamination thickness $d$, coercive force $H_{\mathrm{c}}$, and remanence induction $B_{\mathrm{r}}$, are given in Table I. Steel 1 was investigated experimentally using a computerized measurement system [21] employing Epstein frames. A low-frequency frame (700 turns) was used at magnetizing frequencies lower than $200 \mathrm{~Hz}$, and a high-frequency frame (100 turns) was used at higher frequencies, both conforming with the IEC standard [24].

The static major loop and symmetrical minor loops of Steel 1, shown in Fig. 2(b), were measured using the low-frequency frame connected to a permeameter with the period of controlled sinusoidal induction of the order of 300 seconds. Each static curve was measured after an individual demagnetization carried 


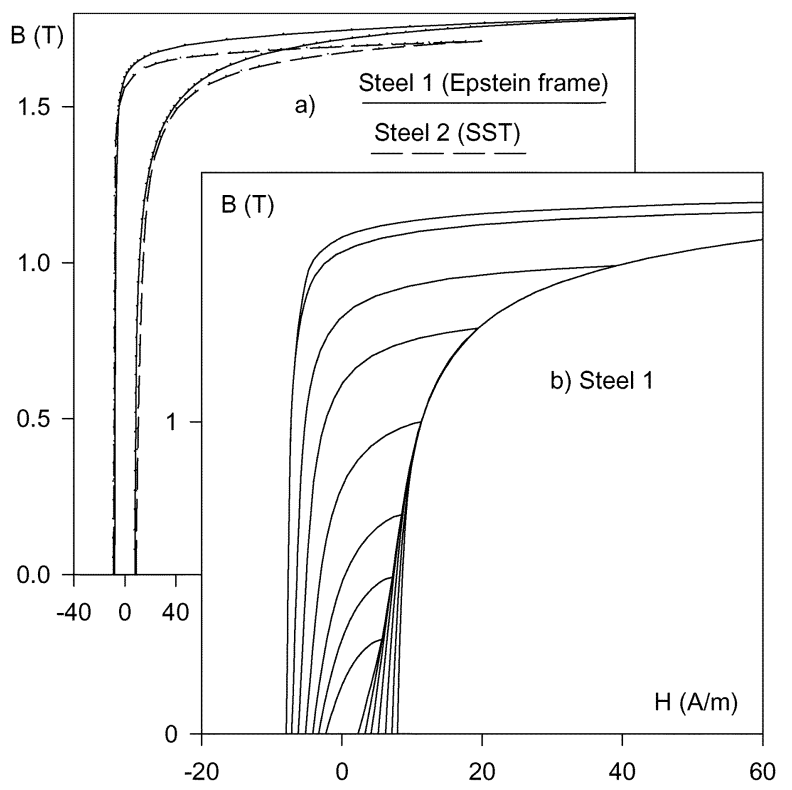

Fig. 2. Static hysteresis curves of Steels 1 and 2.

out with a decreasing alternating voltage. Then the induction was increased to a value $+B_{\mathrm{m}}$ (the movement along the normal magnetization curve was supposed) followed by the induction cycle $+B_{\mathrm{m}},-B_{\mathrm{m}},+B_{\mathrm{m}}$.

The preliminary stage in the measurement of each dynamic magnetization curve was a careful demagnetization at a frequency of $50 \mathrm{~Hz}$ by applying an alternating voltage with its amplitude slowly decreasing from an initial value that was sufficient to cause the sample to approach saturation to zero.

Sheets, $100 \mathrm{~mm}$ by $400 \mathrm{~mm}$, of Steel 2 (30P105 Japanese Industrial Standard) was provided by JFE Steel (Japan) and studied experimentally by means of a double-yoke SST incorporated in a computerized system with controlled sinusoidal induction [25]. The quasi-static centrosymmetric loops were measured at $1 \mathrm{~Hz}$ without waveform control. During the measurement, the fast Fourier transform was used to remove any drift and very high harmonics from $d B / d t$ waveforms. Even harmonics were also removed to symmetrize the waveforms. The inverse Fourier transform was used to reconstruct their time-domain data after time-integration in the frequency domain.

It can be seen from Fig. 2(a) that static major loops of Steels 1 and 2 are almost coincident up to $1.5 \mathrm{~T}$. This allows us to make an indirect comparison between the SST and Epstein frame measurements.

\section{Modeling TeChNiQues}

Both techniques used for the loop calculations by taking into account the effects of eddy currents, are solvers of the equation

$$
\sigma \frac{\partial B}{\partial t}=\frac{\partial^{2} H}{\partial x^{2}} .
$$

This links the magnetic field strength $H(x, t)$ and the magnetic induction $B(x, t)$ in a thin ferromagnetic sheet with conductivity $\sigma=1 / \rho$, where the $x$-axis is normal to the plane of the sheet. A particular solution of (1) for any periodic excitation can be characterized by the steady-state dynamic loop $B_{\mathrm{a}}-H_{\text {sur }}$ (the average magnetic induction versus the surface field) whose area is the total energy $W_{\text {tot }}\left(\mathrm{J} / \mathrm{m}^{3}\right)$ dissipated in unit volume per cycle. If (1) is solved using a static (rate-independent) hysteretic relationship $B_{\mathrm{h}}(H)$, then $W_{\text {tot }}$ contains only hysteretic and eddy-current components easily extracted from $W_{\text {tot }}$.

In order to increase the calculated loop area and thus take into account the excess loss, the hysteresis dependence $B_{\mathrm{h}}(H)$ is represented in the inverse form $H_{\mathrm{h}}(B)$ and supplemented with an external rate-dependent component [18]

$$
H_{\mathrm{exc}}(t)=\delta\left|g(B) \frac{d B(t)}{d t}\right|^{1 / \alpha}
$$

which represents the instantaneous excess field. The exponent $\alpha$ in (2) determines temporal properties of the model (the frequency law of the excess loss simulated by (2) under sinusoidal induction, $\left.W_{\text {exc }} \sim f^{1 / \alpha}[26]\right)$. The function $g(B)$ controls the shape of the dynamic loop. To cover both ascending $(\delta=+1$, $d B / d t>0)$ and descending $(\delta=-1, d B / d t<0)$ trajectories, the induction $B$ in $g(B)$ is always understood as $\delta B$. A common feature of this function for all materials and models studied in the paper is its minimum, $G_{\mathrm{m}}$, at small $|B|$ and its increasing value when approaching positive and negative saturation (this broadens the upper and lower parts of the dynamic loop).

By summing $H_{\mathrm{h}}(B)$ and $H_{\text {exc }}(t)$, we arrive at the DHM [18] which can also be written as

$$
H(t)=H_{\mathrm{h}}(B)+\delta\left[g(B) \frac{d B(t)}{d t} \delta\right]^{1 / \alpha} .
$$

To rewrite (3) in a form suitable for a finite-difference (FD) solver, one should take into account that $H(t)>H_{\mathrm{h}}(B)$ at $\delta=+1$, and $H(t)<H_{\mathrm{h}}(B)$ at $\delta=-1$. Therefore, $[H(t)-$ $\left.H_{\mathrm{h}}(B)\right] \delta=\left|H(t)-H_{\mathrm{h}}(B)\right|$. This equality and the fact that $1 / \delta=\delta$, lead to the expression

$$
\frac{d B}{d t}=\frac{\delta}{g(B)}\left|H(t)-H_{\mathrm{h}}(B)\right|^{\alpha} .
$$

\section{A. Magnetodynamic Models (SHM-S and DHM-S)}

The first numerical technique used in the study is a FD solver of (1) [19] based on the following equation for the internal node $i$ :

$$
\frac{d B_{i}}{d t}=\frac{H_{i-1}-2 H_{i}+H_{i+1}}{\sigma h^{2}} .
$$

Here $H_{i}(t)=H\left(x_{i}, t\right)$ and $B_{i}(t)=B\left(x_{i}, t\right)$ are the grid functions, $h$ is the grid spacing.

The code where $H_{i}(t)$ and $B_{i}(t)$ are linked by the SHM will be referred to as the SHM-solver (SHM-S). With the purpose of taking into account excess loss, (4) is rewritten for the nodal field, $H_{i}$, and nodal induction, $B_{i}$, and the result can be equated with (5), so that

$$
\frac{H_{i-1}-2 H_{i}+H_{i+1}}{\sigma h^{2}}=\frac{\delta_{i}}{g\left(B_{i}\right)}\left|H_{i}(t)-H_{\mathrm{h}, i}\left(B_{i}\right)\right|^{\alpha}
$$

where $H_{\mathrm{h}, i}\left(B_{i}\right)$ is the nodal trajectory, constructed by the SHM. 
TABLE II

MODEL VERSIONS EMPLOYED

\begin{tabular}{|c|c|c|c|}
\hline Model & $\begin{array}{c}\text { Eddy current } \\
\text { field }\end{array}$ & Excess field & Equation \\
\hline DHM-S & FD & Eqs. (2), (4) & $(5),(6)$ \\
\hline SHM-S & FD & None & $(5)$ \\
\hline TSM & $H_{\text {clas }}$ & Eq. (2) & $(8)$ \\
\hline NV-TSM & $H_{\text {clas }}$ & None & $(9)$ \\
\hline
\end{tabular}

In this DHM-solver (DHM-S), system (6) is first solved for $H_{i}(t)$ and then system (5) is integrated in time (both these operations are carried out at every time step).

\section{B. Thin Sheet Model (TSM)}

Under the conditions when the loss separation principle can be applied to the specimen as a whole (this supposes a low enough frequency $f$, or equivalently a thin lamination and uniform flux across its thickness), the loss components can be transformed mathematically into separate field components, so the instantaneous value of the applied field, $H(t)$, is subdivided into hysteresis field, $H_{\mathrm{h}}(t)$, classical eddy-current field, $H_{\text {clas }}(t)$, and excess field, $H_{\text {exc }}(t)$ :

$$
H(t)=H_{\mathrm{h}}(t)+H_{\text {clas }}(t)+H_{\text {exc }}(t) .
$$

Indeed, by multiplying (7) by $d B$ and integrating the product over a cycle, one obtains the energy loss in terms of its components $W=W_{h}+W_{\text {clas }}+W_{\text {exc }}$. Of course it is understood that the components in (7) do not have any independent physical identity beyond the convenience afforded by the mathematical separation.

Using in (7) the well-known formula for $H_{\text {clas }}(t)$ [12] and expression (2), we arrive at the TSM [20]

$$
H(t)=H_{\mathrm{h}}(B)+\frac{d^{2}}{12 \rho} \frac{d B}{d t}+\delta\left|g(B) \frac{d B}{d t}\right|^{1 / \alpha} .
$$

Omitting the last term in (8), a "nonviscous" part of the TSM remains

$$
H(t)=H_{\mathrm{h}}(B)+\frac{d^{2}}{12 \rho} \frac{d B}{d t} .
$$

This is referred to as NV-TSM.

For clarity, the models used in the paper are gathered in Table II.

\section{Static Hysteresis Model (SHM)}

As can be seen from (3), SHM of any type can be used in the composite DHM employed. As in the modeling of NO steels [19], [20], we use the SHM [27] where any reversal curve is constructed by copying and combining some fragments of existing experimental curves used as patterns. Whereas first-order reversal curves were employed as the patterns earlier, in the present study we use, with the same objective, the branches of centrosymmetrical minor loops extended to the major loop tip by means of the normal magnetization curve [28, Fig. 4]. This is, perhaps, the only possible way of reproducing exactly the experimental symmetrical loops (static minor loops) and interpolating between them. This is especially important in modeling

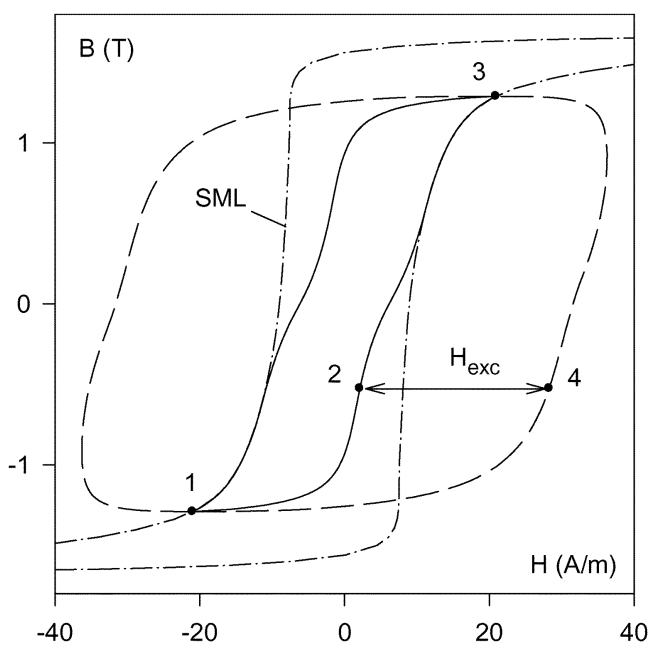

Fig. 3. Static (solid line) and dynamic (dashed line) curves calculated at the surface of Steel $2(200 \mathrm{~Hz})$.

materials such as Steel 2 whose minor loops at low and moderate flux densities tend to be "wasp-waisted" (see loop 1-2-3-1 in Fig. 3).

In the case of oscillating excitation voltage, when $d B / d t$ changes its sign more than twice during the period and asymmetric minor loops appear in the resulting dynamic curve or in the nodal trajectories (for example in the case of pulsewidth modulation (PWM) excitation), the history-dependent version of the SHM (the model with nonlocal memory) should be used. However for "smooth" excitations (sinusoidal, triangle, etc.) a simple history-independent SHM gives the same accurate results if the extended branches of symmetrical minor loops are used for constructing reversal curves (any reversal curve is built by interpolation between them, independent of the magnetization history).

Another advantage of the SHM used in the present work is the simplicity of its inversion [20]. When a reversal curve is constructed at a turning point (for example at point 1 in Fig. 3 ) then the whole static curve 1-2-3 ending at point 3 of the previous reversal is built immediately at the starting point 1 . This curve always lies inside the static major loop (SML) and is kept in the model memory even if the next reversal occurs at some intermediate point 2. Although the curve (1-2-3) is built as $B(H)$-dependence, it is also stored in the inverse form $H_{\mathrm{h}}(B)$ needed for the DHM (4). The independent building of static hysteresis curve (1-2-3) and then dynamic hysteresis curve (1-4-3) enables the excess field $H_{\text {exc }}(t)$ and thus the excess loss to be calculated. At the turning point 3 , where $d B / d t=0$, dynamic curve 1-4-3 concurs with static curve 1-2-3 and new static curve 3-1 is generated.

\section{Model Results}

As the models under study are intended for incorporation into circuit simulators, the main criterion for choosing the model parameters is the best fit to the experimental dynamic loops. It should be noted, however, that the formalization of this criterion is difficult, because the best parameters vary with induction level. Therefore, the model parameters and corresponding 
dynamic loops given in the following two subsections represent a compromise found individually for each material. The reasoning for choosing the model parameters and the discussion of the results obtained are given in corresponding subsections as well as in Section V. To facilitate the comparison of the loops calculated with DHM-S and TSM, they are shown in the left-side and right-side graphs on all the figures below together with corresponding experimental curves in the background. Because of the loop symmetry and to conserve space, all the curves are plotted for positive fields only and the shown ascending loop branches are always implied.

\section{A. Modeling of Steel 1}

In the case of sinusoidally varying magnetic induction, the total loss is often written in the form [12]

$$
W_{\mathrm{tot}}=W_{\mathrm{h}}\left(B_{\mathrm{m}}\right)+\frac{d^{2} \pi^{2} B_{\mathrm{m}}^{2}}{6 \rho} f+C B_{\mathrm{m}}^{1.5} f^{S}
$$

where $S=0.5, W_{\mathrm{h}}\left(B_{\mathrm{m}}\right)$ is determined at $f=0$, and $C$ is a fitting parameter.

It is considered that (10) can be used in the frequency range where the skin effect is negligible. However, there are usually no attempts made to check this condition and evaluate the accuracy of the second term, $W_{\text {clas }}$, in (10). It has been shown in [29] that the values of $W_{\text {clas }}$ for Steel 1 can be considerably different from the eddy-current loss, $W_{\mathrm{ec}}$, found by solving (1) numerically. Thus, already at $f=100 \mathrm{~Hz}$ and for $B_{\mathrm{m}}$ increasing from 0.8 to $1.7 \mathrm{~T}$, the ratio $W_{\text {ec }} / W_{\text {clas }}$ found through the SHM-S increases from 0.85 to 1.26 . Such inaccuracy of $W_{\text {clas }}$ makes it inexpedient to use more complex expression [4] for the excess loss term in (10). Instead, we can consider that any inaccuracy in $W_{\text {clas }}$ is compensated by proper choice of $C$, which should be fitted individually for each $B_{\mathrm{m}}$ [30].

Our attempts to apply (10) to Steel 1 have immediately shown that the exponent $S$ in this equation is also dependent on $B_{\mathrm{m}}$. Therefore, we have first applied (10) individually to each experimental loss curve $\left(B_{\mathrm{m}}=\right.$ const) shown in Fig. 4 in order to observe the change of $S$ with $B_{\mathrm{m}}$. To do so, (10) was considered as a function of two variables $(C$ and $S)$ and fitted in turn to each loss curve in Fig. 4 over the frequency ranges $[0,100]$ $\mathrm{Hz}$ and $[0,200] \mathrm{Hz}$. The dependences $S\left(B_{\mathrm{m}}\right)$ obtained by such least-squares fits are shown in Fig. 5 by dashed and dash-dotted lines, respectively.

Fig. 5 shows that the exponent $S$ in (10) depends on the frequency domain over which the curve fitting is carried out. However, irrespective of the range of fitting, $S>0.5$ at moderate $B_{\mathrm{m}}$ and rapidly decreases at $B_{\mathrm{m}}>1.5 \mathrm{~T}$.

Although (10) is only an approximate formula, dependencies $S\left(B_{\mathrm{m}}\right)$ in Fig. 5 are useful in evaluating the model parameter $\alpha$ (it was shown in [26] that $\alpha=1 / S$ ). In order to identify the DHM used in the DHM-S, these dependencies have been rebuilt into relationships $1 / S$ (Fig. 6) and used as initial values when choosing $\alpha\left(B_{\mathrm{m}}\right)$. Due to the complex interrelation between eddy current and excess loss components calculated through the DHM-S, the adjustment of $\alpha$ requires numerical experiments.

1) DHM-S (When Applied to Steel 1): Calculations carried out through the DHM-S show that at moderate $B_{\mathrm{m}}$ the values of

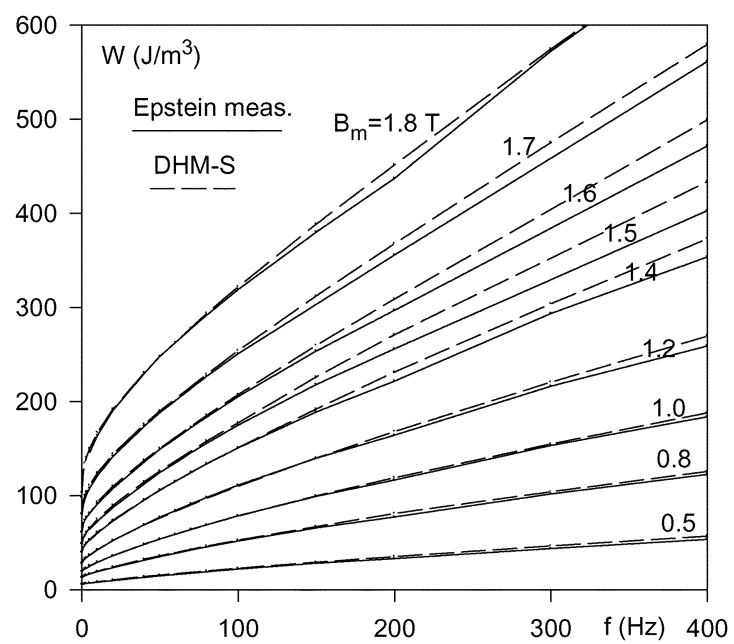

Fig. 4. Losses in Steel 1 measured at sinusoidal magnetic induction (solid lines) and the losses predicted by the DHM-S (dashed lines).

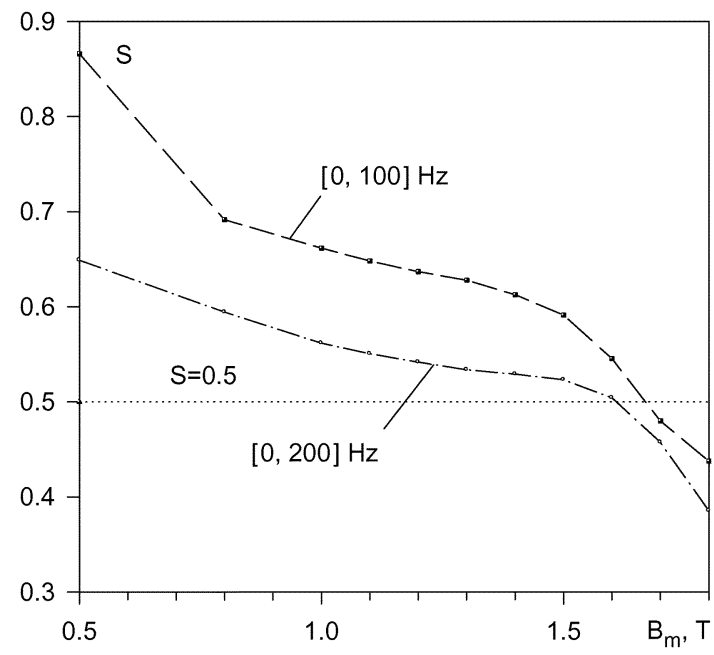

Fig. 5. Variation of $S$ with $B_{\mathrm{m}}$ found by least-squares fit of the measured losses in Fig. 4 by means of (10).

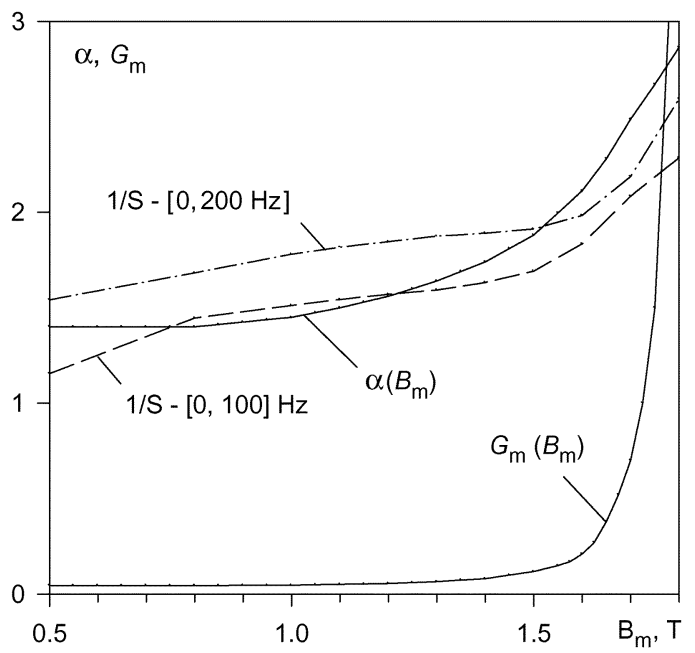

Fig. 6. Dependencies $\alpha\left(B_{\mathrm{m}}\right)$ and $G_{\mathrm{m}}\left(B_{\mathrm{m}}\right)$ used in modeling Steel 1.

a are closer to the lower curve $1 / S$ in Fig. 6 . As $B_{\mathrm{m}}$ increases, the fitted dependence $\alpha\left(B_{\mathrm{m}}\right)$ approaches the upper curve $1 / S$ 

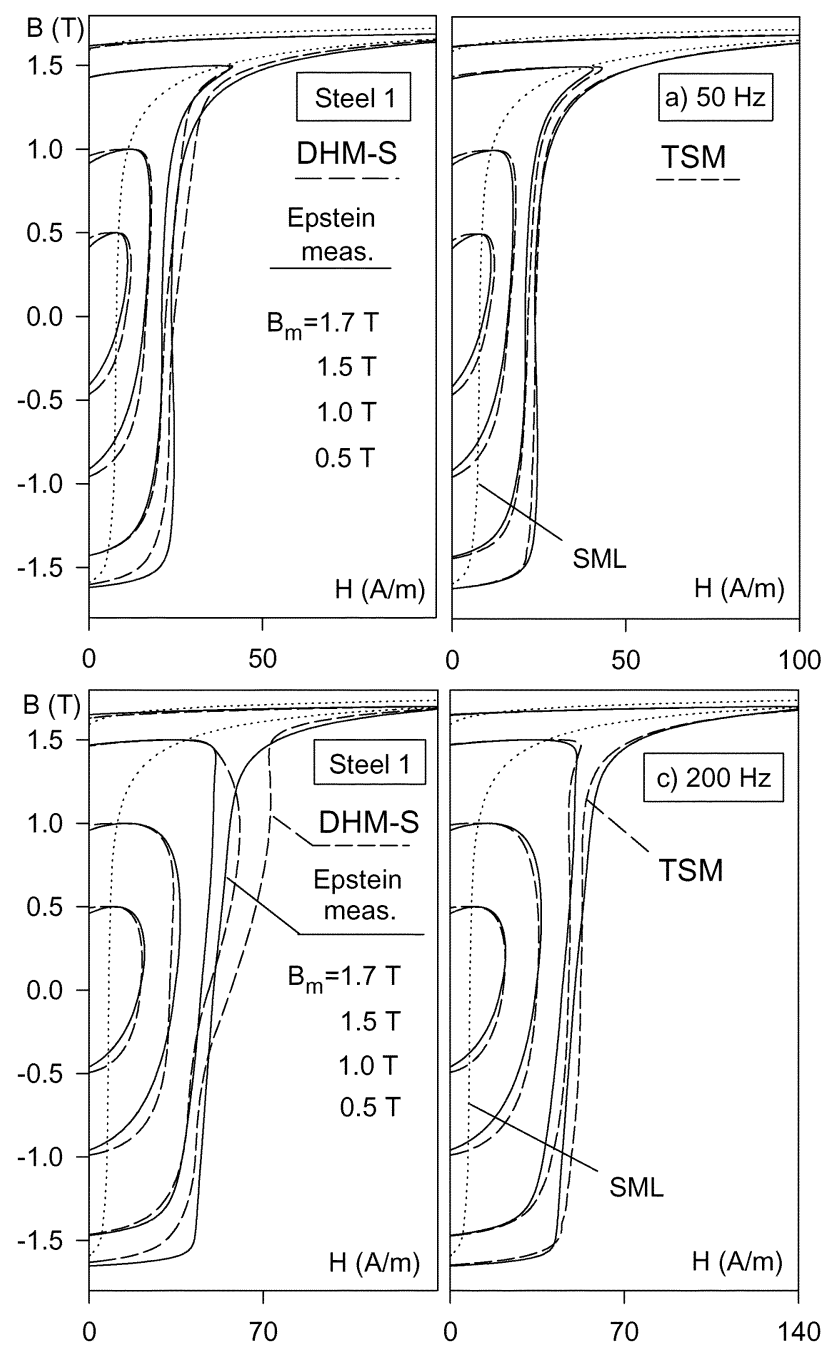
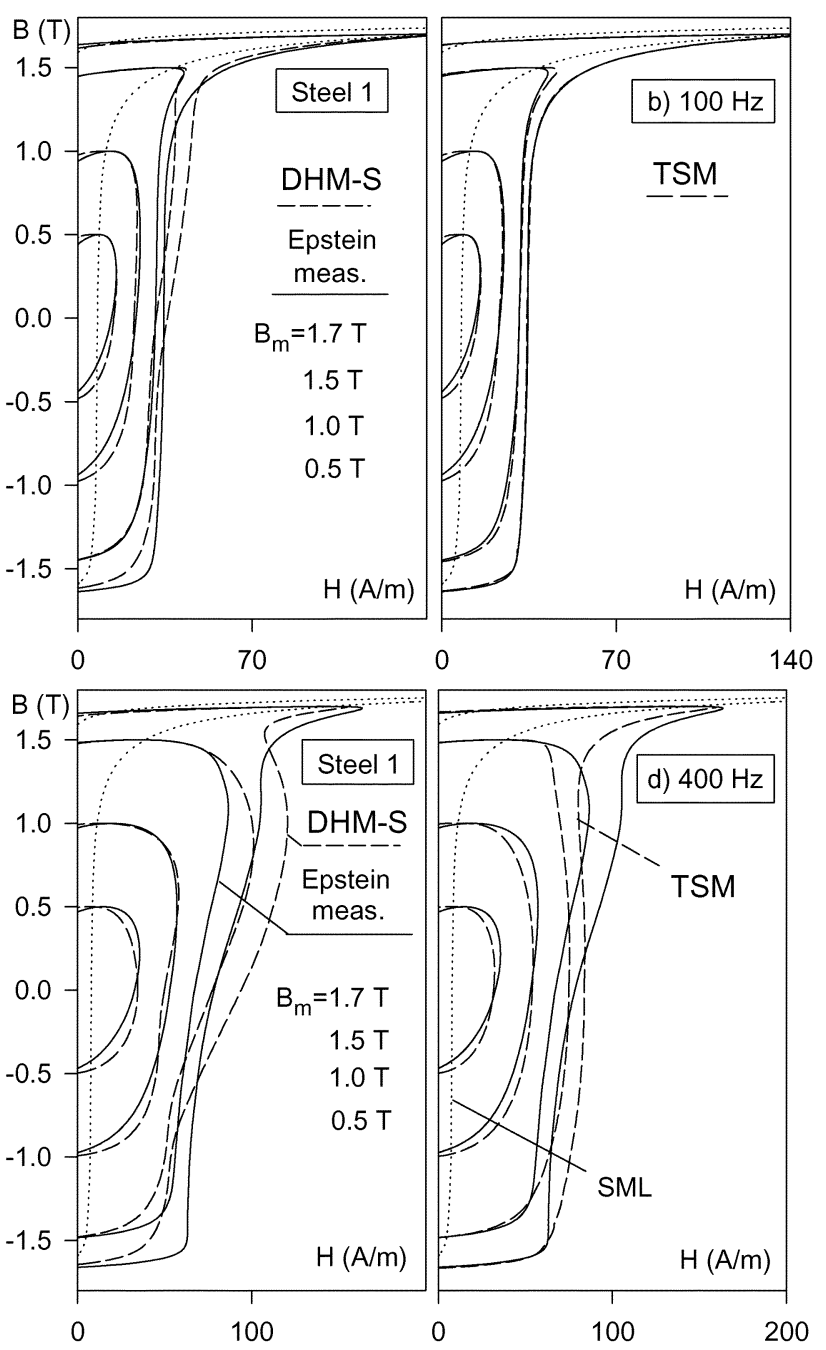

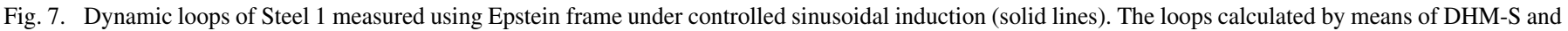
TSM are shown by dashed lines in the left-side and right-side figures, respectively.

and then exceeds it. Dependence $\alpha\left(B_{\mathrm{m}}\right)$ in Fig. 6 was built simultaneously with the relationship $G_{\mathrm{m}}\left(B_{\mathrm{m}}\right)$ whose values were chosen so as to equalize the calculated and measured losses at $100 \mathrm{~Hz}$.

The following sequences for calculating parameters of the DHM-S have been found acceptable for Steel 1:

$$
g= \begin{cases}G_{\mathrm{m}} & \text { if } 0.3 \leq B \leq 0.5, \\ G_{\mathrm{m}}+0.4 G_{\mathrm{m}}(0.3-B) & \text { if } B<0.3, \\ G_{\mathrm{m}}+0.8 G_{\mathrm{m}}(B-0.5)^{2} & \text { if } B>0.5\end{cases}
$$

The relationships $\alpha\left(B_{\mathrm{m}}\right)$ and $G_{\mathrm{m}}\left(B_{\mathrm{m}}\right)$ in Fig. 6 have been described by linear splines and used in the calculations under arbitrary voltage excitation. Since the DHM-S is a time-stepping solver which does not "know" in advance the induction waveforms in different sheet layers (FD nodes), we cannot use the relationships shown in Fig. 6 directly. However, at any reversal static and dynamic nodal trajectories coincide (this behavior was shown in Fig. 3) and the distance $\Delta B$ between current and previous turning points is determined. The value of $B_{\mathrm{m}}$ can then be set equal to $\Delta B / 2$ which allows one to calculate $\alpha\left(B_{\mathrm{m}}\right)$ and $G_{\mathrm{m}}\left(B_{\mathrm{m}}\right)$ (these values are kept fixed until the next nodal reversal).
Dynamic loops predicted by this version of the DHM-S under sinusoidal flux densities are shown in Fig. 7 (left-side graphs). Corresponding loss curves are represented in Fig. 4 by dashed lines. They show that at higher $B_{\mathrm{m}}$ the values of a could be even greater than those used in the model (this would entail a greater curvature of the loss dependencies). However, this can result in poorer loop shapes.

A more demanding test of the model is the verification of its predictive ability under nonsinusoidal voltage waveforms. The measured and calculated loops are shown in Figs. 8-10.

2) TSM (When Applied to Steel 1): The similar technique of varying $G_{\mathrm{m}}$ and $\alpha$ has been employed when choosing parameters of the TSM. In an attempt to reproduce the total loss behavior over the frequency range $0-200 \mathrm{~Hz}$ values $\alpha=2.0$ and $G_{\mathrm{m}}=0.152$ at $B_{\mathrm{m}}<1.6 \mathrm{~T}$ have been chosen. As expected from the previous results, at higher $B_{\mathrm{m}}$ both $\alpha$ and $G_{\mathrm{m}}$ should increase. In particular, at $B_{\mathrm{m}}=1.8 \mathrm{~T}$ the best value of $\alpha$ which is 2.5 , corresponds to $W_{\text {exc }} \sim f^{0.4}$. The following formulas were found acceptable to calculate model parameters at $B_{\mathrm{m}}>1.6 \mathrm{~T}$ :

$$
\begin{aligned}
\alpha & =2+4.197\left(B_{\mathrm{m}}-1.6\right)^{1.322} \\
G_{\mathrm{m}} & =0.152+73.23\left(B_{\mathrm{m}}-1.6\right)^{2.692} .
\end{aligned}
$$




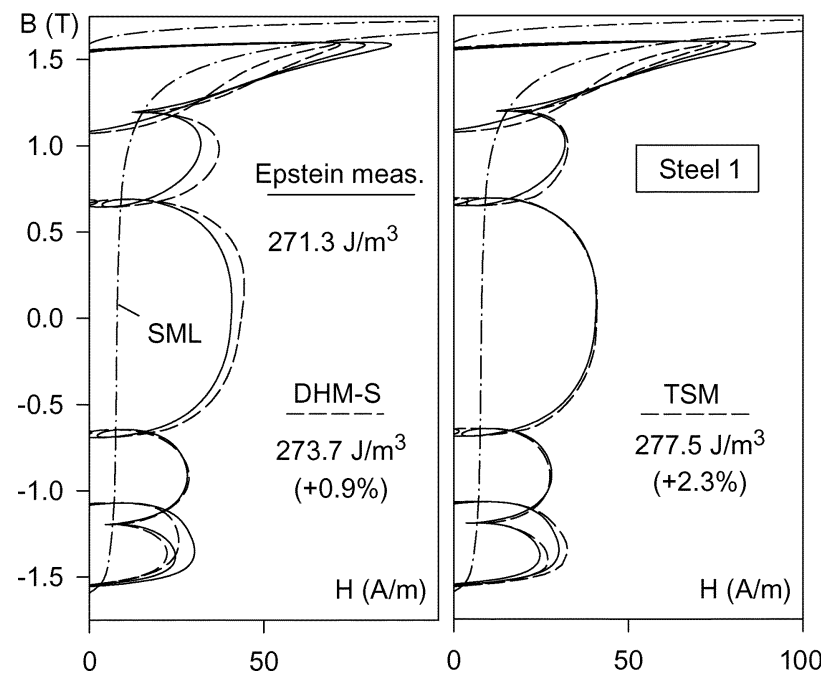

Fig. 8. Measured and calculated loops under the voltage which contains fundamental $(50 \mathrm{~Hz}), 3 \mathrm{rd}, 7 \mathrm{th}$, and 11th harmonics.

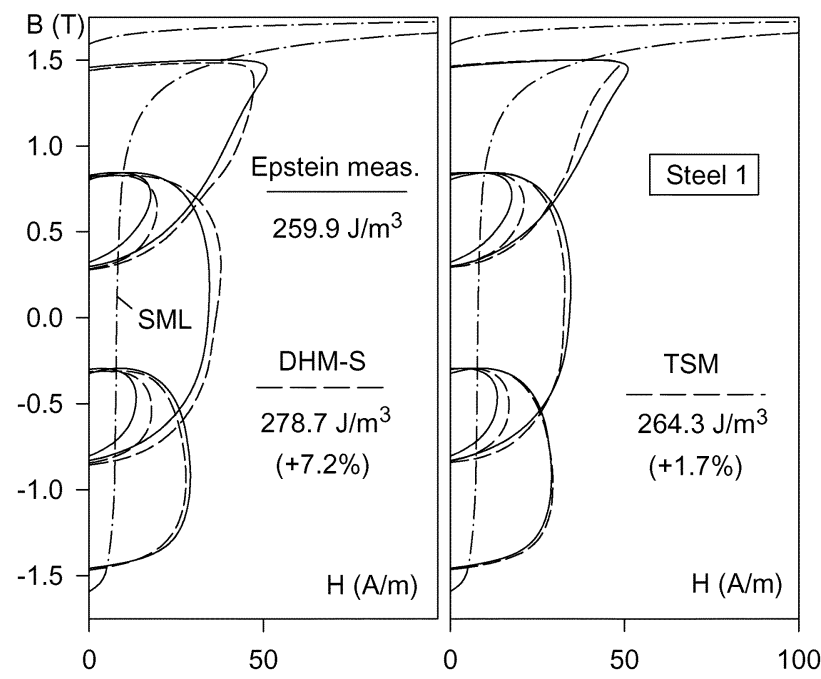

Fig. 9. Measured and calculated loops under the voltage which contains fundamental $(50 \mathrm{~Hz})$ and 5th harmonic $(50 \%)$.

To reproduce the shape of the dynamic loops, the following computational chain was used in the TSM regardless of the value of $G_{\mathrm{m}}$ chosen:

$$
g= \begin{cases}\mathrm{g}_{1}=G_{\mathrm{m}} /\left[1-(B / 1.9)^{2}\right], & \text { if } B>0, \\ g_{2}=g_{1}+0.2 G_{\mathrm{m}} B & \text { if } B<0, \\ g_{3}=g_{1}-0.1 G_{\mathrm{m}} B & \text { if } B<-1, \\ g_{4}=g_{3}+0.8 G_{\mathrm{m}}(-1-B) & \text { if } B<-1.2, \\ g_{5}=g_{4}+2 G_{\mathrm{m}}(-1.2-B) & \text { if } B<-1.4, \\ g_{6}=g_{5}+8 G_{\mathrm{m}}(-1.4-B) & \text { if } B<-1.5 .\end{cases}
$$

In this way, it is possible to vary $\alpha$ and $G_{\mathrm{m}}$ depending on the loop height and vary $g$ with $B(t)$.

Dynamic loops predicted by the TSM under sinusoidal flux densities are shown in Fig. 7 (right-side graphs). Corresponding total losses are represented in Fig. 11 (dashed lines). As can be seen in Fig. 11, the value $\alpha=2.0$ gives a somewhat overestimated total loss at $f<50 \mathrm{~Hz}$ and $B_{\mathrm{m}}=1.5 \mathrm{~T}$.
At higher $f$ and $B_{\mathrm{m}}$, the TSM prediction is accurate. The same remarkable agreement between the TSM predictions and experiment is observed in the case of nonsinusoidal induction (right-side graphs in Figs. 8-10).

It is interesting to compare the losses calculated by the TSM described by (12) and (13) with the losses obtained by the same model but using fixed values of $\alpha$ and $g$. In order to reproduce the "typical" excess loss dependence $W_{\text {exc }} \sim f^{0.5}$, we again put $\alpha=2$ and choose $g=G_{\mathrm{m}}=$ const so as to obtain equal calculated and measured total loss at $B_{\mathrm{m}}=1.5 \mathrm{~T}, f=100 \mathrm{~Hz}$ (point $C$ in Fig. 12). As shown by dashed curves in Fig. 12, such a simplified model gives the same inadequate loss figures as those obtained by the statistical loss theory [4, Fig. 12]. In particular, the losses calculated at $B_{\mathrm{m}}<1.5 \mathrm{~T}$ are slightly higher than those measured, whereas the losses calculated at $B_{\mathrm{m}}>1.5 \mathrm{~T}$ are markedly underestimated.

\section{B. Modeling of Steel 2}

1) DHM-S (When Applied to Steel 2): As with Steel 1, Steel 2 demonstrates different frequency dependence of the excess loss at low and high inductions. It was found acceptable to keep $\alpha=$ 1.5 and $g=G_{\mathrm{m}}=0.08$ if the distance $\Delta B$ between turning points is less than $2.8 \mathrm{~T}$ and if these points have opposite signs of $B$. Otherwise, parameters of the DHM-S were described by the following routine:

$$
\begin{gathered}
\alpha=1.8, \quad G_{\mathrm{m}}=0.15, \quad g_{1}=G_{\mathrm{m}} /\left[1-(B / 2)^{2}\right], \\
g= \begin{cases}\mathrm{g}_{2}=g_{1}+5 G_{\mathrm{m}}(B-1)^{2} & \text { if } B>1, \\
\mathrm{~g}_{3}=g_{1}-0.3 G_{\mathrm{m}} B & \text { if } B<0, \\
\mathrm{~g}_{4}=g_{3}+G_{\mathrm{m}}(-1-B) & \text { if } B<-1, \\
\mathrm{~g}_{5}=g_{4}+6 G_{\mathrm{m}}(-1.3-B) & \text { if } B<-1.3, \\
\mathrm{~g}_{6}=g_{5}+8 G_{\mathrm{m}}(-1.4-B) & \text { if } B<-1.4, \\
\mathrm{~g}_{7}=g_{6}+26 G_{\mathrm{m}}(-1.5-B) & \text { if } B<-1.5 .\end{cases}
\end{gathered}
$$

As $\Delta B$, and therefore $\alpha$ and $G_{\mathrm{m}}$, are calculated individually for each FD node, and since the resulting dynamic induction is the average of the nodal inductions $B_{i}$, no smooth transition between $\alpha$ and $G_{\mathrm{m}}$, calculated for small and large $\Delta B$ was used in the code.

Dynamic loops predicted by the DHM-S under sinusoidal flux densities are shown in Fig. 13 (left-side graphs). Frequency variation of corresponding total losses is represented in Fig. 14 in comparison with the measured losses. The loss curves shown in Fig. 14 pass through the measured points with somewhat different peak inductions. For instance, the curve $B_{\mathrm{m}}=1.7 \mathrm{~T}$ connects the points with $B_{\mathrm{m}}=1.705,1.708,1.689$, and 1.682 $\mathrm{T}$. These values as well as the peak inductions for other loss curves are given in Fig. 13 (left-side graphs).

The loop and total loss calculated through the DHM under a nonsinusoidal induction [23] (Fig. 15, left-side) coincides well with the measured loop and loss.

2) TSM (When Applied to Steel 2): When applying the TSM to Steel 2 magnetized under sinusoidal induction, it was necessary to implement a smooth transition from $\alpha=1.5$, which characterizes the steel at $B_{\max }=1.02 \mathrm{~T}$, to $\alpha=1.8$ corresponding to $B_{\mathrm{m}}=1.7 \mathrm{~T}$. Then, using one experimental loop as a pattern, for example loop $\mathrm{P}$ in Fig. 16(a) $(f=50 \mathrm{~Hz}$, 

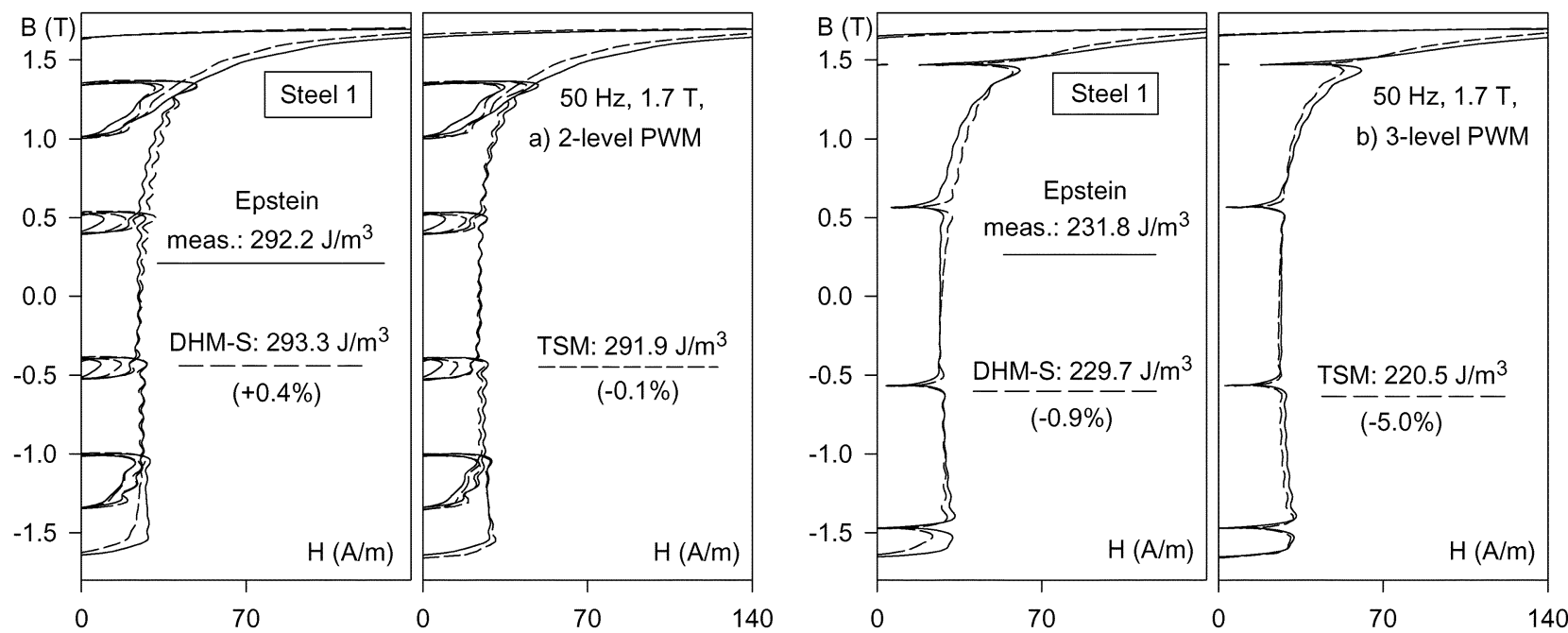

Fig. 10. Measured and calculated loops under (a) 2-level and (b) 3-level PWM voltage which contains 9 pulses per cycle $(f=50 \mathrm{~Hz}, B \mathrm{~m}=1.7 \mathrm{~T})$.

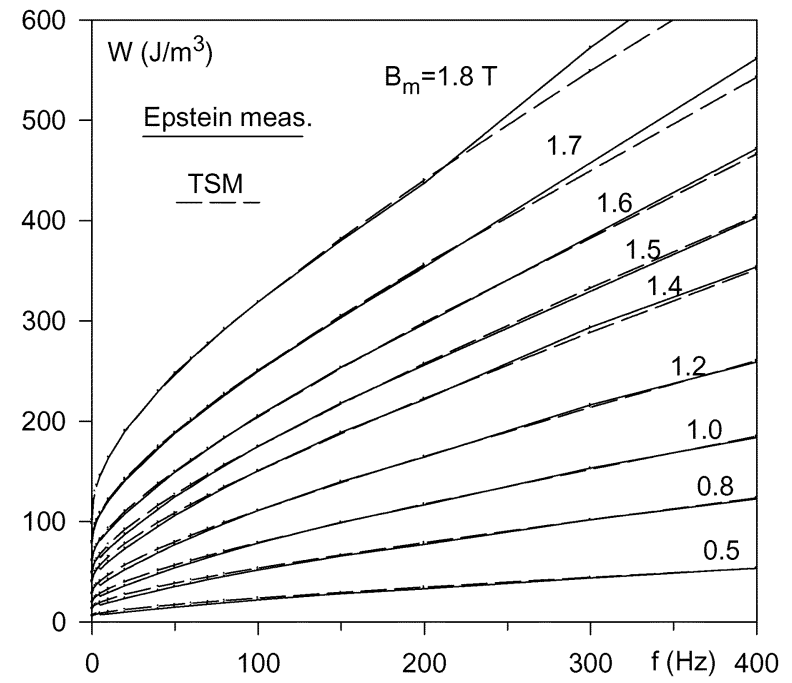

Fig. 11. Loss characteristics of Steel 1 calculated by the TSM (12), (13) (dashed lines) compared to the measured losses (solid lines).

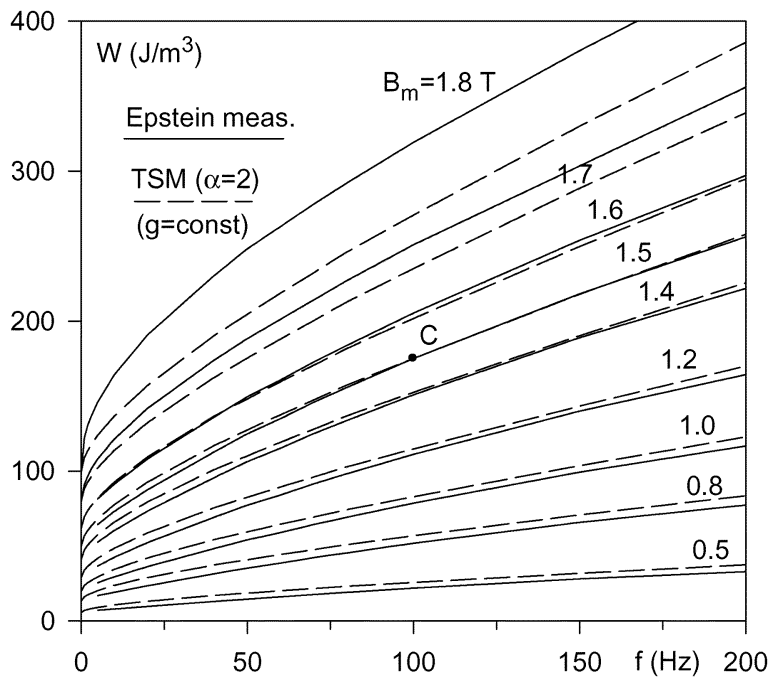

Fig. 12. Loss characteristics calculated by the TSM with $\alpha=2$ and fixed $g=G_{\mathrm{m}}=0.214$ (dashed lines) compared to the measured losses (solid lines).

$B_{\mathrm{m}}=1.705 \mathrm{~T}$ ), function $g(B)$ can be found by taking the analytical form of the TSM (8). For this purpose, it is sufficient to build the loop NV by means of the NV-TSM, and calculate the excess field $H_{\text {exc }}$ as the difference in $H$ between the curves P and NV. In accordance with (2), the calculated field $H_{\text {exc }}$ is a function of $B(t)$ and $\mathrm{d} B / \mathrm{d} t$ which were also used in the NV-TSM. Using these values in (2) and solving it for $g(B)$, we can represent $g(B)$ by a pointwise approximated function $E(B)$. An idea of the graph of $E(B)$ can be obtained by plotting the difference between the curves NV and $\mathrm{P}$ in Fig. 16(a) as a function of $B$. The smooth increase of $\alpha$ and $g$ with $\Delta B$ is written as the following sequence:

$$
\begin{aligned}
& B_{\mathrm{m}}=\Delta B / 2 ; \quad a=1.5 ; \quad g=0.5 E(B) \\
& \left\{\begin{array}{l}
\text { if } B_{\mathrm{m}}>1.021 \text { then } \\
\alpha=1.5+0.4931\left(B_{\mathrm{m}}-1.021\right)^{1.2643} \\
g=E(B)\left[0.5+0.9606\left(B_{\mathrm{m}}-1.021\right)^{1.6612}\right]
\end{array}\right.
\end{aligned}
$$

The loops calculated by the TSM under sinusoidal and nonsinusoidal flux densities are shown in the right-side plots of Figs. 13 and 15, respectively.

\section{DISCUSSION OF RESULTS}

A general characteristic of all the loops calculated at sinusoidal flux densities by the DHM-S and TSM is a very good agreement between theory and experiment at peak induction levels lower than 1.0-1.3 T, and somewhat poorer accuracy of both the models at higher levels. In particular, marked convex segments appear in the upper parts of the ascending branches calculated by the DHM-S as the peak induction increases above $1.3 \mathrm{~T}$. This feature is especially pronounced in Steel 1 studied using Epstein frame. On the contrary, the TSM-calculated ascending branches are flatter which makes the TSM inadequate at higher frequencies.

Since both models are developed within the framework of the classical approach, it is expedient to search first for explanations of these features through the analysis of the conventional solutions of (1), i.e., solutions obtained by the SHM-S (they can also be obtained by the DHM-S with a negligibly small viscosity factor $G_{\mathrm{m}}$ ). Examples are represented in Fig. 17, where classically calculated loops of Steel $1\left(B_{\mathrm{m}}=1.0\right.$ and $\left.1.7 \mathrm{~T}\right)$ are compared with corresponding loops measured at sinusoidal 

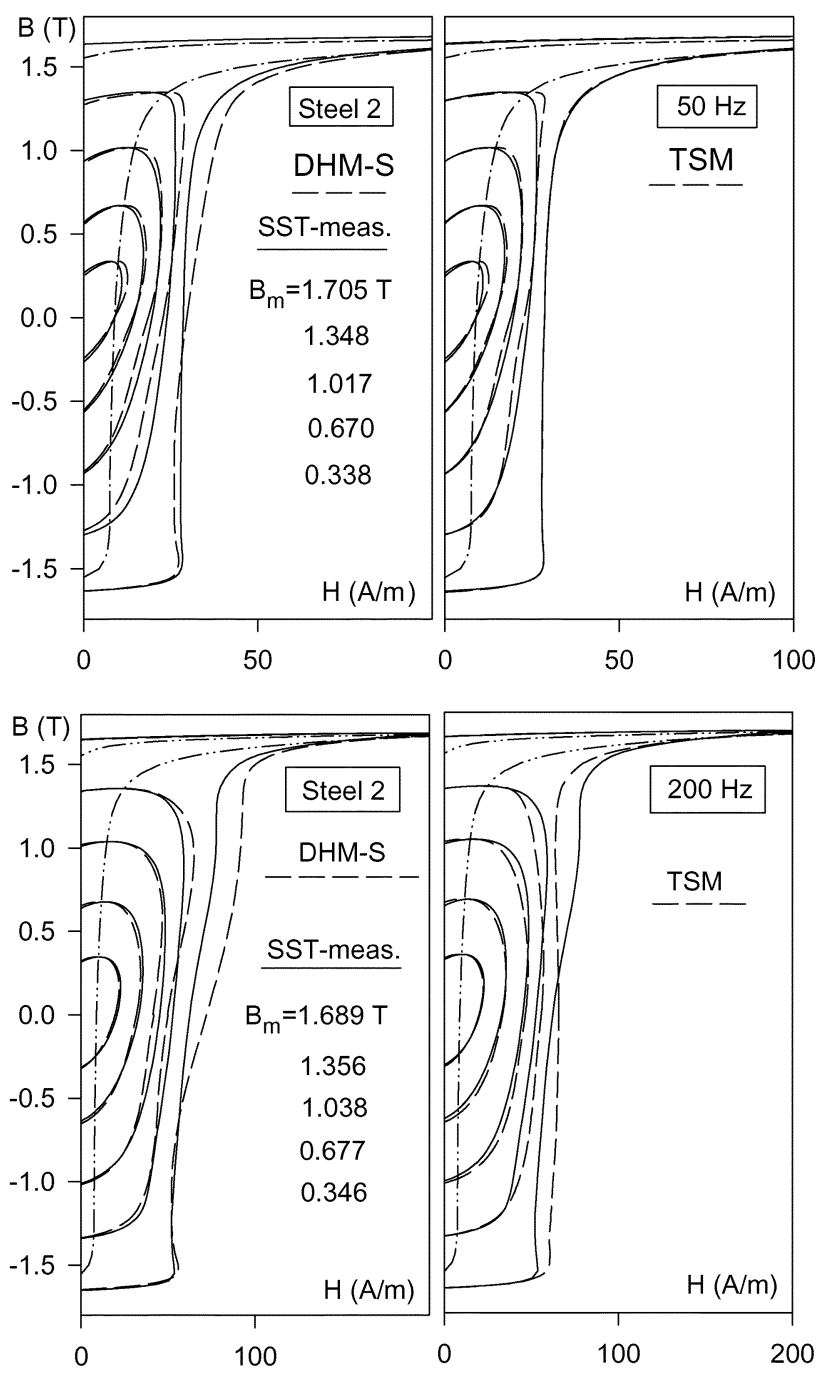
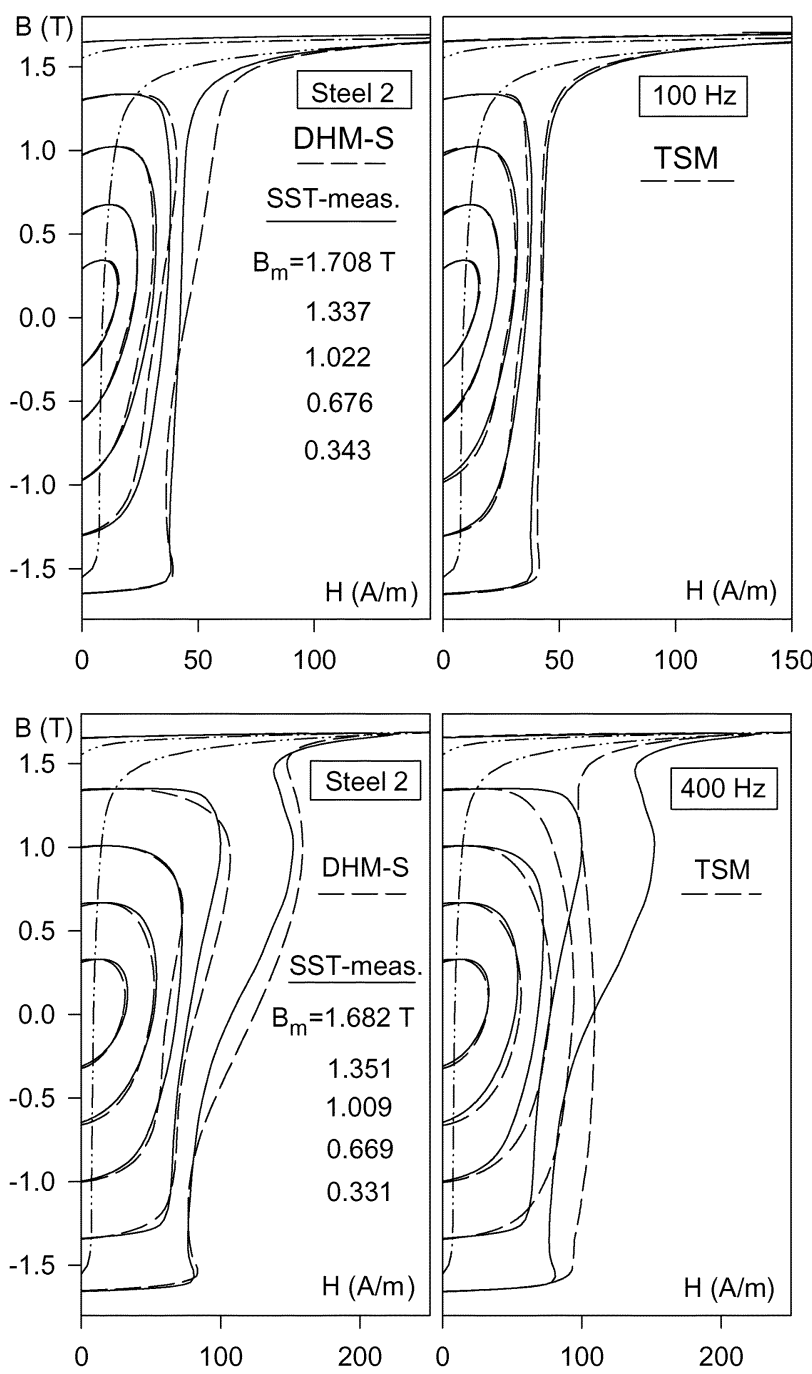

Fig. 13. Dynamic loops of Steel 2 measured with the SST under controlled sinusoidal induction (solid lines). The loops calculated by means of DHM-S and TSM are shown by dashed lines in the left-side and right-side figures, respectively.

flux densities. As can be seen in Fig. 17, there is a substantial difference between the shape and position of the calculated and experimental loops. Thus, the loops calculated at $B_{\mathrm{m}}=1.0 \mathrm{~T}$ lie completely inside the measured loops, and there is enough empty space in $H$ between them to fill it with the excess field component. On the contrary, the loops calculated at $B_{\mathrm{m}}=1.7 \mathrm{~T}$ almost touch the measured loops at point $\mathrm{C}$. This means that the introduction of any dynamic component in the hysteresis model will result in the loops exceeding the bounds of experimental loops. At the same time, the large distance between the lower parts of calculated and measured ascending branches (the distance A-B) explicitly requires the introduction of the dynamic term in (3) and causes its dependence on $B$ implemented in the DHM (3) by means of the function $g(B)$. As a general rule, $g(B)$ should increase when approaching positive and, especially, negative saturation and can be nearly constant at lower $|B|$ (the latter is desirable to reproduce the loops at small and moderate peak induction).

Unfortunately it is impossible to obtain a fully controlled excess field $H_{\text {exc }}$ through the DHM-S. In particular, we cannot nullify the excess field at point $C$ (Fig. 17). Because of the phase shift between flux densities in different sheet layers, there is no direct analogy between the shape of $g(B)$ and the change of the resulting excess field with $B$. The convex segments appearing in the loops calculated through the SHM-S are kept in the loops obtained through the DHM-S that causes the difference between calculations and measurements in both the loop shapes and losses. In principle, the latter difference can be reduced by decreasing $g(B)$ at large negative $B$, but this leads to poorer modeling of the "heel" at the bottom of the measured ascending branches (i.e. the loop segments about points B in Fig. 17).

It is rather unexpected that over the frequency range 0-200 $\mathrm{Hz}$ better modeled loops can be obtained for both the steels by means of the TSM (8). It is interesting that the higher accuracy of the TSM is reached due to the error of its component $H_{\text {clas }}(t)$. The change of this error with frequency and peak induction can be seen in Fig. 16, where the loops calculated by the NV-TSM (9) are compared to the loops calculated by the SHM-S (the latter can be described as a nonviscous version of the DHM-S). Fig. 16(a) shows that the difference between the loops calculated by the NV-TSM and SHM-S appears already 

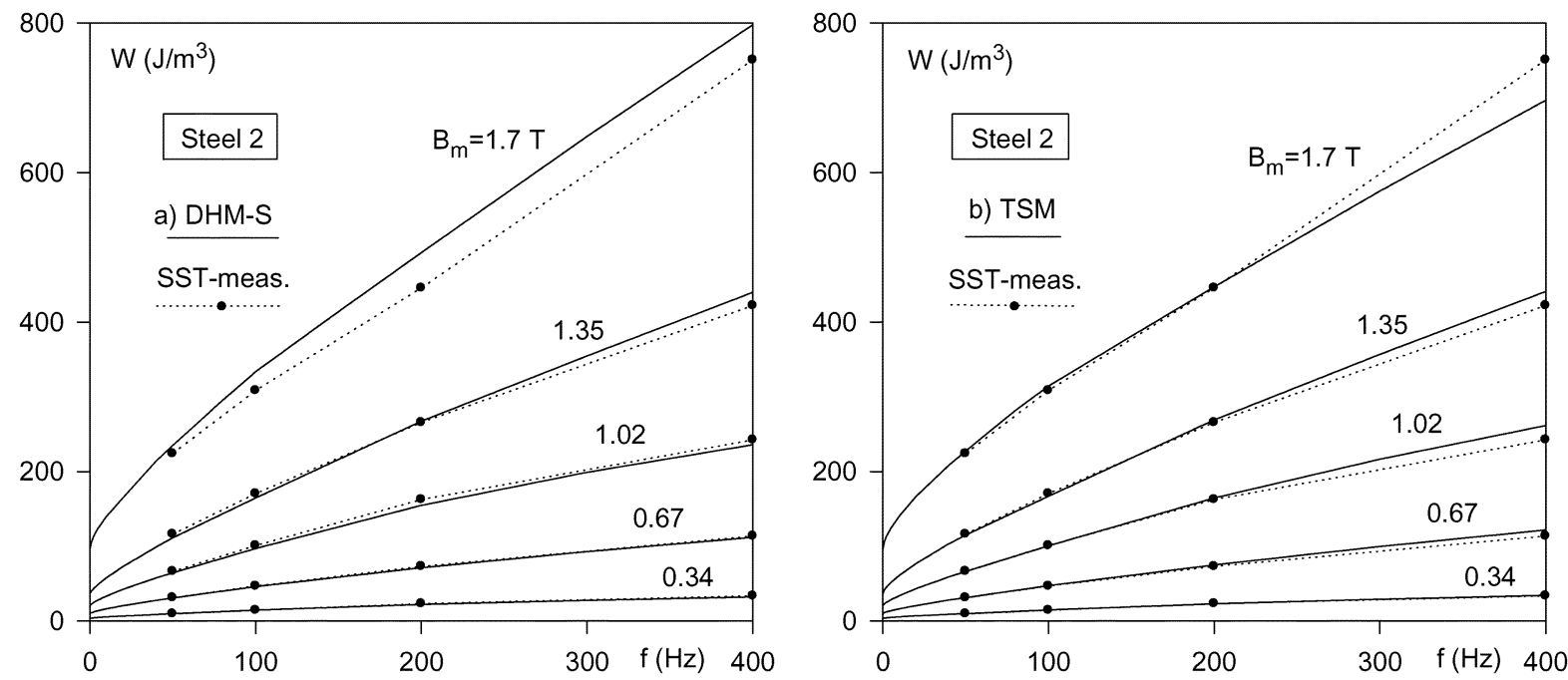

Fig. 14. Losses calculated by the DHM-S (a) and TSM (b) (solid lines) in comparison to the losses measured by the SST (dotted lines).

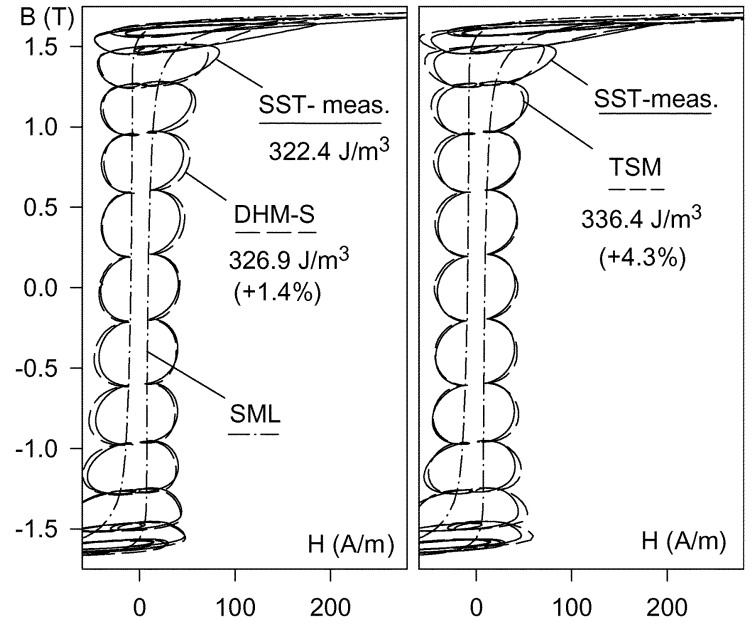

Fig. 15. Measured and calculated loops of Steel 2 under nonsinusoidal voltage [23].

at $50 \mathrm{~Hz}$ for the loops at $B_{\mathrm{m}}=1.7 \mathrm{~T}$. At $100 \mathrm{~Hz}$ the distortions of the NV-TSM-loops become more pronounced, and at higher frequencies these loops lose any similarity with the loops found by the SHM-S. However, an advantage of the NV-TSM-loops is the absence of the convex segments in the upper parts of their ascending branches and the presence of such segments in the lower parts. The former reserves a space for the excess field and avoids undesirable convexity in the loops calculated by the TSM and thus stops them crossing measured loops when introducing viscosity term in the model. The latter facilitates modeling the "heels" of the measured ascending branches for those with $B_{\mathrm{m}}$ of $1.7 \mathrm{~T}$.

Using Figs. 16(c), (d) and 17(a), (b) the subject of the comparison of the loops taken from Epstein frame and SST can be touched upon. Attention is mainly focused on the loops measured at maximum peak induction, $1.7 \mathrm{~T}$ in this particular case, where according to [22] the difference between Epstein- and SST-measured losses is maximum.

The remarkable feature of the loops taken from the SST (Fig. 16) are the convex segments, which reproduce the seg-
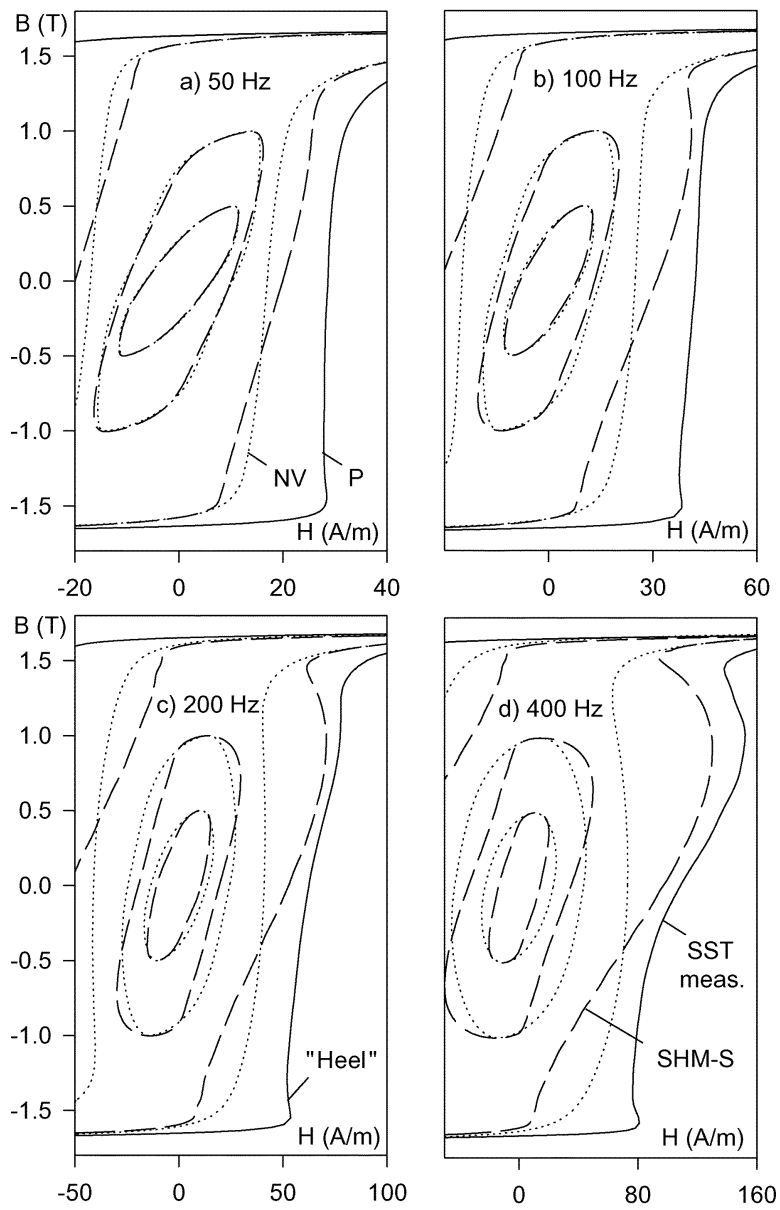

Fig. 16. Dynamic loops calculated for Steel $2\left(B_{\mathrm{m}}=0.5,1.0\right.$, and $\left.1.7 \mathrm{~T}\right)$ through the NV-TSM (dotted lines) and through the SHM-S (dashed lines). Solid lines are the loops taken from the $\operatorname{SST}\left(B_{\mathrm{m}}=1.7 \mathrm{~T}\right)$.

ments predicted by the SHM-S. These convexities are then reproduced by the DHM-S that corroborates the applicability of the generalized classical method to sheets of GO steel. At the same time, the absence of the convex segments in the loops taken from the Epstein frame and the difficulties in modeling 

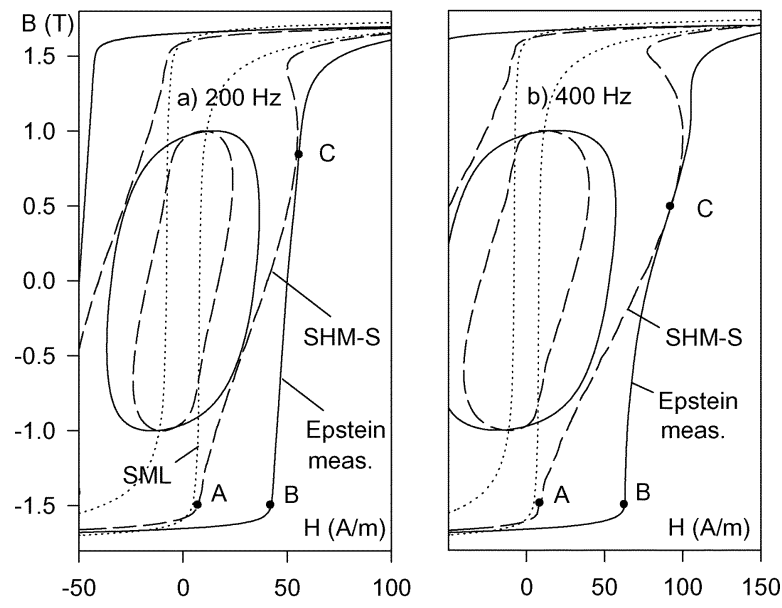

Fig. 17. Comparison of dynamic loops of Steel 1 calculated by the SHM-S at $B_{\mathrm{m}}=1.0$ and $1.7 \mathrm{~T}$ (dashed lines) with experimental loops (solid lines).

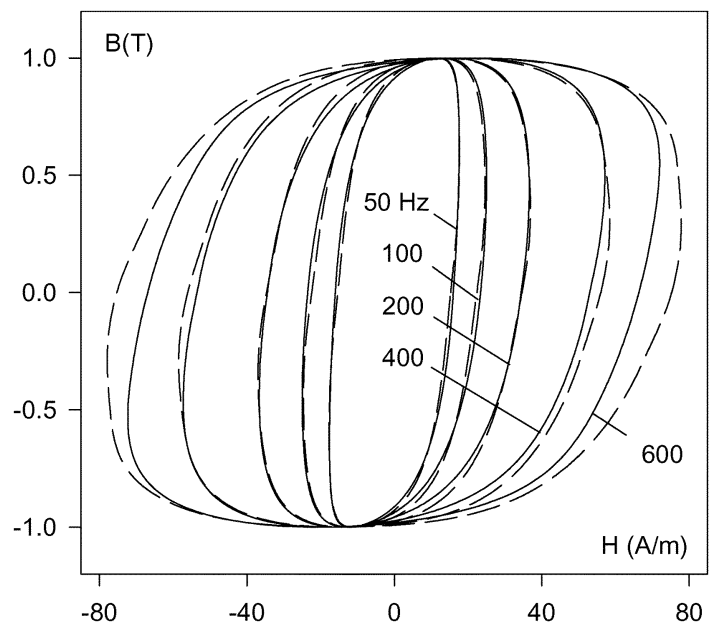

Fig. 18. Comparison of dynamic loops of Steel $1\left(B_{\mathrm{m}}=1 \mathrm{~T}\right)$ calculated by the DHM-S (dashed lines) and measured at $f=50,100,200,400$, and $600 \mathrm{~Hz}$ (solid lines).

these loops at higher induction can be a result of inconsistency of the thin single sheet modeled and the measuring fixture, which should be considered, in the case of anisotropic steel, as a 3-D construction.

\section{MAGNETIC DOMAIN INTERPRETATION}

A general observation made above is that the DHM-S is able to predict dynamic loops very accurately in the induction region lower than 1.0-1.3 T, and that it works well enough at higher induction levels. This accuracy is somewhat unexpected because it has been obtained by means of a 1-D model applied to the material, which is usually described by 2-D calculation schemes. To explain the model's ability, it should be first noted that its accuracy decreases with increasing induction and frequency. It is instructive, in this respect, to observe the frequency evolution of the loops whose peak inductions are far from saturation and which were modeled very accurately up to $400 \mathrm{~Hz}$. The loops of Steel 1 calculated and measured at $B_{\mathrm{m}}=1 \mathrm{~T}$ and $f=50,100,200,400$, and $600 \mathrm{~Hz}$ are shown in Fig. 18 .

A visible difference between the model and experiment appears only at $400 \mathrm{~Hz}$ when the calculated loop has $3.7 \%$ greater
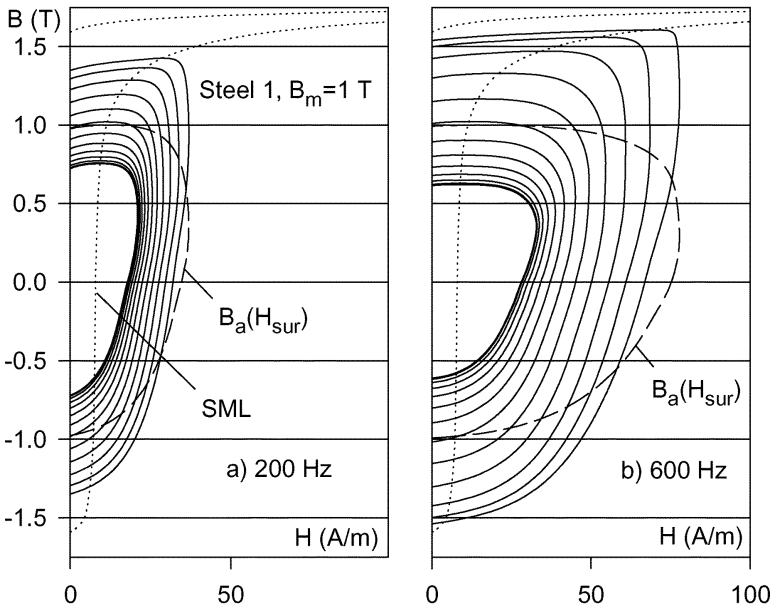

Fig. 19. Nodal (solid lines) and resulting (dashed lines) loops calculated for Steel 1 at $B_{\mathrm{m}}=1 \mathrm{~T}$ and $f=200 \mathrm{~Hz}$ (a) and $f=600 \mathrm{~Hz}$ (b).

area than the measured loop. At $600 \mathrm{~Hz}$, this difference reaches 9.5\%. It is noticeable that at lower frequencies (see Fig. 7) similar discrepancies are observed only at higher inductions $\left(B_{\mathrm{m}}=\right.$ 1.5-1.7 T). This leads to the suggestion that the errors in the model calculation appear as the flux density in some sheet layers exceeds an induction level $B_{\text {rot }}$ above which domain rotation becomes the dominant magnetization mechanism. By convention it is considered [31] that this level is at about the "knee" of static major loop (for Steel $1 B_{\mathrm{rot}} \approx 1.5 \mathrm{~T}$ ). This assumption can be verified by comparing Fig. 19(a) and (b), where dynamic loops calculated at $B_{\mathrm{m}}=1 \mathrm{~T}(200$ and $600 \mathrm{~Hz}$, respectively) are shown together with nodal $B_{i}-H_{i}$ trajectories for the odd nodes of the FD grid employed. It should be remembered that the induction $B$ of the resulting dynamic $B-H$ loop is calculated by averaging the inductions $B_{i}(t)$ of these trajectories. The incidental detail in Fig. 19 is that the nodal trajectories lie outside the static major loop showing that calculations were made by means of the DHM. As seen in Fig. 19(b) $(f=600 \mathrm{~Hz})$, some nodal trajectories exceed the level $B_{\text {rot }}$, whereas in Fig. 19(a) $(f=200 \mathrm{~Hz})$ all these trajectories lie within this level $\left(\left|B_{i}\right|<B_{\text {rot }}\right)$.

Returning to the major theme of 2-D medium-1-D model, the contradictions between the classical and domain schemes in Fig. 1 should be recalled. Characteristic features of the 1-D classical solution are the same nodal induction $B_{i}(t)=B\left(x_{i}, t\right)$ for the whole sheet layer at the depth $x_{\mathrm{i}}$ (it is shown conventionally in Fig. 1(a)), and the change of the local induction with depth. On the contrary, the local induction in the domain structure shown in Fig. 1(b) changes from domain to domain, but is the same at any depth. These contradictions are eliminated if the well-known idea about the DW bowing during the course of the magnetization is used.

In this case, any $B_{i}(t)$ calculated with 1-D classical model can be regarded as a value obtained by averaging $B$-values over all "positive" and "negative" domains at the depth of the $i$ th grid node (i.e., within a conventionally narrow sheet layer shown in Fig. 20 by horizontal dashed lines).

Taking into account the above assumption that magnetization by the DW displacement is mainly completed at $|B|=B_{\text {rot }}$, 


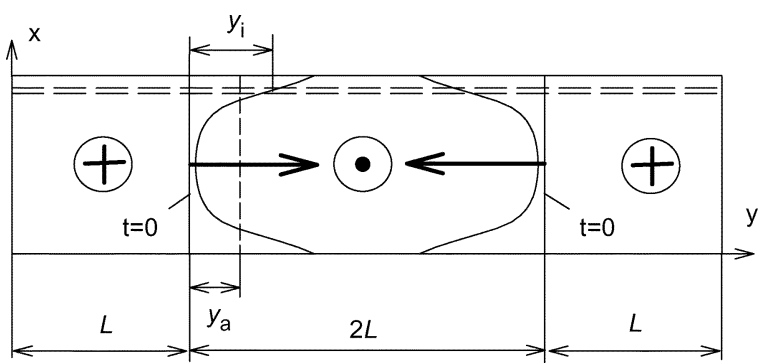

Fig. 20. A segment of a sheet cross section (the vertical frame lines are lines of mirror symmetry in a cross section of infinite width).

we should consider for the purposes of this model calculation that the induction within individual domains is $\pm B_{\text {rot }}$. This allows us to restore the DW shape and observe its evolution in time. Assuming an infinite regular domain structure with DW spacing $2 L$ at equilibrium, it is sufficient to analyze any sheet segment of width $2 L$ (or $4 L$ as in Fig. 20). Since the calculation begins after a quasi-static demagnetization and the initial induction at all the nodes is the same $\left(B_{i}=0\right)$, such a segment is characterized by vertical domain walls labeled as $t=0$. The different $B_{i}(t)$ during the course of magnetization determine a curved DW profile at a given instant of time. (The point of the wall situated at the level of the $i$ th node is determined by the coordinate $\left.y_{i}(t)=L B_{i}(t) / B_{\text {rot }}\right)$.

If the material is excited by a cosinusoidal voltage, then the flux density averaged over the whole sheet cross section varies sinusoidally and the mean wall position with respect to equilibrium (vertical dashed line in Fig. 20) is determined as

$$
y_{\mathrm{a}}(t)=\left(L B_{\mathrm{m}} / B_{\text {rot }}\right) \sin (2 \pi f t)
$$

(this formula is given in [2], where saturation induction $B_{\mathrm{s}}$ is used instead of $B_{\text {rot }}$ ).

The evolution of the DW during three stages of the magnetization process is shown in Fig. 21 as a sequence of DW profiles at instants separated by equal time intervals $(\Delta t=T / 20)$. During the initial stage, Fig. 21(a), the average induction, $B(t)$, increases from 0 to $+B_{\mathrm{m}}$. The arrows show that magnetization occurs due to the widening of the positive domains at the expense of negative ones. During the second stage, Fig. 21(b), $B(t)$ decreases from $+B_{\mathrm{m}}$ to $-B_{\mathrm{m}}$, and Fig. 21(c) corresponds to the third stage when $B(t)$ increases from $-B_{\mathrm{m}}$ to $+B_{\mathrm{m}}$. At the end of this stage, at $t=1.25 \mathrm{~T}$, a steady-state regime is established and DW profiles change in accordance with Fig. 21(b) and (c) by turns.

Illustrative DW profiles in Fig. 21 were constructed for $B_{\mathrm{m}}=$ $1 \mathrm{~T}, f=200 \mathrm{~Hz}$, and $2 L / d=3$. Based on the same solution, similar plots can also be constructed for any other $2 L / d$, including the average wall spacing of a given material. In this case, they should be similar to the bowing DW profiles obtained by Bishop [32] for the magnetization model based on the DW displacement but without domain rotation. It should be noted, however, that unlike Bishop's approach, the wall bowing diagrams above are simply a way of plotting the $B_{z}(x)$ predicted by 1-D model. The 2-D domain structures depicted play no active role in the calculations. Nevertheless, they reproduce qualitatively all characteristic features of DW behavior including the rise of
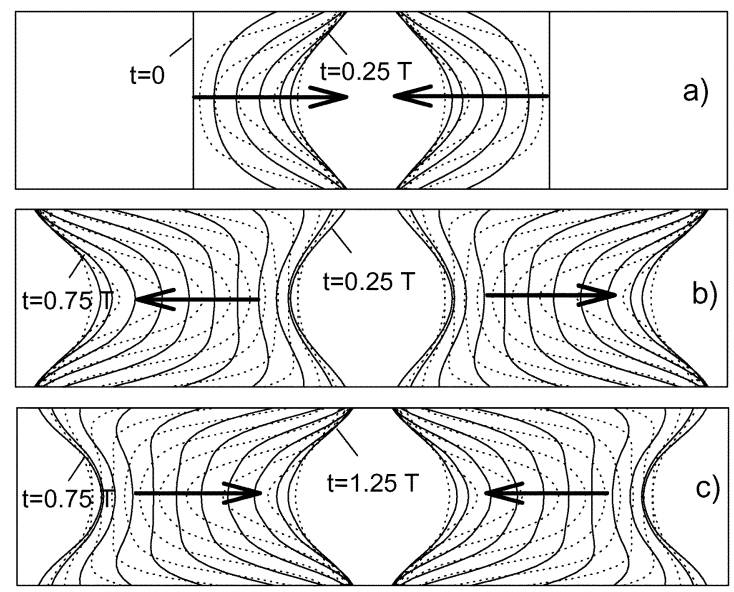

Fig. 21. Evolution of domain walls starting from the demagnetized state. DW profiles calculated with the DHM-S and SHM-S are shown by solid and dashed curves, respectively.

wall bowing with frequency and the inverse proportionality between the DW bowing and total loss [12, p. 419], [32]. The latter phenomenon is illustrated by dotted DW profiles in Fig. 21 calculated with zero viscosity function $g(B)$. These walls are much more bowed and may be regarded as being completely flexible. This means that increasing $g(B)$ leads to the widening of the dynamic loop and to the flattening of the bowed DWs.

The DW representation above can be useful in explanation of the limitation of the 1-D model employed. As was shown above, the model works very accurately as long as $\left|B_{i}\right|<B_{\text {rot }}$. At higher nodal flux densities (due to the skin effect they can be reached even at moderate average induction) wall bowing becomes so severe that walls meet at the sheet surface forming a surface domain. Beginning from this moment and ending at the time when the surface domain disappears during the subsequent stage of the magnetizing process, (16) can no longer be used since domain rotation becomes dominant at surface layers of the sheet. It is natural to expect that different model parameters or even different models should be used in modeling the loops formed when either wall displacement or domain rotation are predominant. This means that if the model is adjusted accurately to experimental loops of low and moderate peak inductions $B_{\mathrm{m}}$, then its ability can be somewhat poorer in predicting the loops (and losses) at high $B_{\mathrm{m}}$ and/or high magnetization frequency when the assumption about DWs as well-defined geometrical surfaces becomes questionable [12, p. 420]. As shown in Section IV this is typical for the two steels, which exhibit quite different frequency behavior at low and high induction levels.

Differences between predicted and measured loss can be due to the erroneous, universally applied assumption that the magnetic path length is fixed at $0.92 \mathrm{~m}$ as specified in [24]. In practice the magnetic path length varies with several factors. For example, it drops by $4 \%-5 \%$ in GO steel when $B_{\mathrm{m}}>1.7 \mathrm{~T}$ and increases by around 3\% when $f$ is increased from 50 to 400 $\mathrm{Hz}$ [33]. This should be taken into account to make the difference between the true (absolute) loss and the measured loss independent of $B_{\mathrm{m}}$ and $f$ but, since this is not done in practice for Epstein or SST measurements, care must be applied when 
assessing the accuracy of predicted losses at different flux densities or magnetizing frequencies.

\section{CONCLUSION}

The possibilities of a modified classical approach in modeling dynamic hysteresis loops and the prediction of the energy loss in GO electrical steels has been presented. The essence of the modification is the introduction of a time delay of the magnetic induction behind the magnetic field implemented in the solver of the penetration equation (DHM-S) and in the simplified model (TSM) applied to a bulk material. Particular attention has been devoted to the frequency dependence of the excess loss component which is usually written as a term $C f^{S}$ with $S=0.5$. By combining accurate measurements and numerical experiments, we have found that the exponent $S$ decreases from about 0.6 to 0.7 at low to moderate inductions to about 0.3 to 0.4 at high flux densities. This shows that probably different mechanisms determine excess losses at different peak inductions. The good performance of the TSM up to $200 \mathrm{~Hz}$ is explained by the error in the simplified (low frequency) formula for the classical field. To explain the predictive ability of the DHM-solver, the results obtained through this model have been interpreted in terms of domain walls with bowing profiles. This interpretation does not involve any specific mechanisms in calculation so it should be viewed only as a possible explanation of the applicability of 1-D solver to 2-D calculation scheme of the modeled medium. Both the interpretation and the loss separation are somewhat artificial in the case of GO materials. Even if the statistical explanation [12] of the loss separation is accepted, it is difficult to agree with the evaluation of the classical loss by the formula corresponding to a nondomain medium (the second term in (10)). Of particular interest in this respect is that the accuracy of the model is highest in the prediction of small symmetrical loops, when domain walls make small excursions from equilibrium, and some aspects of the statistical theory are not applicable [12]. Although both models employed are, strictly speaking, phenomenological, their usage is justifiable in the absence of a rigorous physical theory and comparatively accurate prediction of hysteresis loops and losses under sinusoidal and nonsinusoidal conditions. At the same time, this approach might not be as accurate as in the case of NO steel [19], [20]. This is due to the different domain structures in GO and NO materials. Before attempting to improve the models, it is desirable to gain a better insight into experimental results and differentiate between the errors of the model itself and the influence of 3-D experimental fixture. An important topic to back up future work in this direction is clarification of the effect of measuring system geometry on loss values by a systematic comparison of different Epstein frames [33] and parallel experiments on Epstein frame and SST.

\section{ACKNOWLEDGMENT}

S. E. Zirka is indebted to Dr. J. Bishop (Sheffield University, U.K.) for useful discussion on the paper during 2003-2004.

The experimental part of the work was supported by EPSRC Grant EP/C518616/1, which also provided Visiting Fellowships for Prof. S. E. Zirka to spend time at the Wolfson Centre for Magnetics, Cardiff University, U.K.

\section{REFERENCES}

[1] K. H. Stewart, "Losses in electrical sheet steel," J. IEE, vol. 97, pt. II, pp. $121-125,1950$.

[2] R. H. Pry and C. P. Bean, "Calculation of the energy loss in magnetic sheet materials using a domain model," J. Appl. Phys., vol. 29, pp. 532-533, 1958.

[3] J. E. L. Bishop, "Enhanced eddy current loss due to domain displacement," J. Magn. Magn. Mater., vol. 49, pp. 241-249, 1985.

[4] G. Bertotti, "General properties of power losses in soft ferromagnetic materials," IEEE Trans. Magn., vol. 24, no. 1, pp. 621-630, Jan. 1988.

[5] M. Celasco, A. Mazoero, P. Mazzetti, and A. Stepanescu, "Study of Bloch wall dynamic and losses in monocrystalline, polycrystalline and amorphous materials by means of optical techniques," J. Appl. Phys., vol. 57, no. 1, pp. 4238-4243, 1985.

[6] F. N. Dunaev, "On the energy losses in the magnetization of ferromagnets," Phys. Met. Met., vol. 29, no. 5, pt. I, pp. 937-946, 1970.

[7] P. Mazzetti, "Bloch walls correlation and magnetic loss in ferromagnets," IEEE Trans. Magn., vol. MAG-14, no. 5, pp. 758-763, Sep. 1978

[8] D. C. Jiles and D. L. Atherton, "Theory of ferromagnetic hysteresis," J. Magn. Magn. Mater., vol. 6, no. 1, 2, pp. 48-60, 1986.

[9] H. Pfutzner, J. Bishop, and G. Harasko, "A multi-parametric domain model for highly grain-oriented polycrystalline Si-Fe," J. Magn. Magn. Mater., vol. 133, pp. 49-52, 1994.

[10] D. C. Jiles, "Dynamics of domain magnetization and the Barkhausen effect," Czechoslovak J. Phys., vol. 50, pp. 893-988, 2000.

[11] H. Hauser, D. C. Jiles, Y. Melikhov, L. Li, and R. Grossinger, "An approach to modeling the dependence of magnetization on magnetic field in the high field regime," J. Magn. Magn. Mater., vol. 300, pp. 273-283, 2006.

[12] G. Bertotti, Hysteresis in Magnetism. San Diego, CA: Academic, 1998.

[13] G. Bertotti, "Dynamic generalization of the scalar Preisach model of hysteresis," IEEE Trans. Magn., vol. 28, no. 5, pp. 2599-2601, Sep. 1992.

[14] L. R. Dupre, G. Bertotti, V. Basso, F. Fiorillo, and J. Melkebeek, "Generalisation of the dynamic Preisach model toward grain oriented $\mathrm{Fe}-\mathrm{Si}$ alloys," Physica-B, vol. 275/1-3, pp. 202-206, 2000.

[15] S. E. Zirka, Y. I. Moroz, P. Marketos, and A. J. Moses, "Properties of dynamic Preisach models," Physica-B, vol. 343/1-4, pp. 85-89, 2004.

[16] L. R. Dupre, O. Bottauscio, M. Chiampi, M. Repetto, and J. A. A Melkebeek, "Modeling of electromagnetic phenomena in soft magnetic materials under unidirectional time periodic flux excitations," IEEE Trans. Magn., vol. 35, no. 5, pp. 4171-4184, Sep. 1999.

[17] D. Makaveev, L. Dupre, M. De Wulf, and J. Melkebeek, "Dynamic hysteresis modelling using feed-forward neural networks," J. Magn. Magn. Mater., vol. 254-255, pp. 256-258, 2003.

[18] S. E. Zirka, Y. I. Moroz, P. Marketos, and A. J. Moses, "Dynamic hysteresis modeling," Physica-B, vol. 343/1-4, pp. 90-95, 2004.

[19] S. E. Zirka, Y. I. Moroz, P. Marketos, and A. J. Moses, "Viscositybased magnetodynamic model of soft magnetic materials," IEEE Trans. Magn., vol. 42, no. 9, pp. 2121-2132, Sep. 2006.

[20] S. E. Zirka, Y. I. Moroz, P. Marketos, A. J. Moses, and D. C. Jiles, "Measurement and modeling of $B-H$ loops and losses of high silicon non-oriented steels," IEEE Trans. Magn., vol. 42, no. 10, pp. 3177-3179, Oct. 2006.

[21] S. Zurek, P. Marketos, T. Meydan, and A. J. Moses, "Use of novel adaptive digital feedback for magnetic measurements under controlled magnetizing conditions," IEEE Trans. Magn., vol. 41, no. 11, pp. 4242-4249, Nov. 2005.

[22] J. Sievert, "The measurement of magnetic properties of electrical sheet steel-Survey on methods and situation of standards," J. Magn. Magn. Mater., vol. 215-216, pp. 647-651, 2000.

[23] T. Matsuo and M. Shimasaki, "Simple modeling of the AC hysteretic property of a grain-oriented silicon steel sheet," IEEE Trans. Magn, vol. 42, no. 4, pp. 919-922, 2006.

[24] Methods of Measurement of the Magnetic Properties of Electrical Steel Sheet and Strip by Means of an Epstein Frame, IEC 60404-2.

[25] T. Nakase, M. Nakano, K. Fujiwara, and N. Takahashi, "Method of digital waveform control for measuring magnetic properties by means of a single sheet tester," Trans. IEE Jpn., vol. 119-A, pp. 1019-1025, July 1999. 
[26] S. E. Zirka, Y. I. Moroz, P. Marketos, and A. J. Moses, "A viscoustype dynamic hysteresis model as a tool of loss separation in conducting ferromagnetic laminations," IEEE Trans. Magn, vol. 41, no. 3, pp. 1109-1111, Mar. 2005.

[27] S. E. Zirka, Y. I. Moroz, P. Marketos, and A. J. Moses, "Congruencybased hysteresis models for transient simulations," IEEE Trans. Magn., vol. 40, no. 2, pp. 390-399, Mar. 2004.

[28] K. Zakrzewski and F. Pietras, "Method of calculating the electromagnetic field and power losses in ferromagnetic materials, taking into account magnetic hysteresis," Proc. IEE, vol. 118, no. 11, pp. 1679-1685, Nov. 1971.

[29] S. E. Zirka, Y. I. Moroz, P. Marketos, and A. J. Moses, "Evolution of the loss components in ferromagnetic laminations with induction level and frequency," J. Magn. Magn. Mater., 2008, accepted for publication.

[30] E. Barbisio, F. Fiorillo, and C. Ragusa, "Predicting loss in magnetic steels under arbitrary induction waveform and with minor hysteresis loops," IEEE Trans. Magn., vol. 40, no. 4, pp. 1810-1819, 2004.
[31] R. M. Bozorth, Ferromagnetism. New York: Van Nostrand, 1951.

[32] J. E. L. Bishop, "The analysis of eddy-current-limited magnetic domain wall motion, including severe bowing and merging," J. Phys. D: Appl. Phys., vol. 6, pp. 97-115, 1973.

[33] P. Marketos, S. Zurek, and A. J. Moses, "A method for defining the mean path length of the Epstein frame," IEEE Trans. Magn., vol. 43, no. 6, pp. 2755-2757, 2007.

[34] F. N. Dunaev, "On the energy losses in the magnetization of ferromagnets," Phys. Met. Met., Part 2, vol. 30, no. 2, pp. 666-668, 1970.

[35] F. N. Dunaev, "On the energy losses in the magnetization of ferromagnets," Phys. Met. Met., Part 3, vol. 32, no. 3, pp. 633-635, 1971.

Manuscript received July 18, 2007; revised February 28, 2008 and May 2, 2008. Published August 20, 2008 (projected). Corresponding author: P. Marketos (e-mail: marketosp@cf.ac.uk). 